Supporting information for the paper:

\title{
Molecular Rotors: Design, Synthesis, Structural Analysis and Silver Complex of New [7.7]Cyclophanes
}

by

Niculina Bogdan, Guillaume Benoît, Ion Grosu, Loïc Toupet, Yvan Ramondenc, Eric Condamine, Ioan Silaghi-Dumitrescu and Gérard Plé

\section{Table of contents}

1. Synthesis of compounds 2-5 2

2. Experimental determination of the barriers of rotation for compounds 2-5. 3

3. Molecular modeling for compounds $2 \mathrm{a}-5 \mathrm{a} \quad 4$

2.1 Geometry optimizations 4

2.2. Barriers of rotation $\quad 7$

4. X-ray Crystalographic Data 10

5. Experimental section $\quad 12$

5.1. General experimental data 12

$\begin{array}{ll}5.2 \text { General procedure for the synthesis of diol } 7 & 12\end{array}$

5.3 General procedure for the preparation of the [7.7]cyclophanes $2 \mathrm{a}-5 \mathrm{a}$ and [7.7.7.7] cyclophane $4 b \quad 13$

5.4 General procedure for synthesis of complex 6a 13

$\begin{array}{ll}\text { 5.5. Compounds characterization } & 14\end{array}$

5.6 Copies of ${ }^{1} \mathrm{H}$ and ${ }^{13} \mathrm{C}$ NMR spectra of compounds 2-7. 20

5.7 Parameters of the crystalographic determinations for compound 2a, 5a, 6a. 27

5.8 Cartesian coordinates of the B3lyp/6-31G(d) optimized structures of $2 \mathrm{a}-5 \mathrm{a} \quad 29$ 


\section{Synthesis of compounds $2-5$.}

The synthesis of the new macrocyclic cyclophanes (2-5) was performed reacting the cis,cis isomer of compound 7 with isomeric phtaloyl dichlorides and the dichloride of 2,6-pyridine dicarboxylic acid (Scheme 1), using DMAP as base, and acetonitrile as solvent, by a typical procedure for the synthesis of macrocyclic esters. ${ }^{1,2}$ The reactions underwent in fair to good yields (31-52\%) with the obtaining of monomers (main products) and dimers (side products observed for 2 and 4). All monomers (2a-5a; $n=1$, Scheme 1) and the dimer $\mathbf{4 b}(n=2$, scheme 1) were separated by flash-chromatography or by crystallization and were investigated as single compounds. The compound 7 is easily obtained by ketalization reaction ${ }^{3}$ of 2,6diacetylpyridine with 2-hydroxymethyl-2-methyl-1,3-propanediol. The compound 7 is obtained as a mixture of isomers: cis,cis; cis,trans and trans,trans in a ratio of 1.1/1.8/1 (78 \% yield). The cis,cis isomer was separated by flash chromatography in a correct yield (16\%).

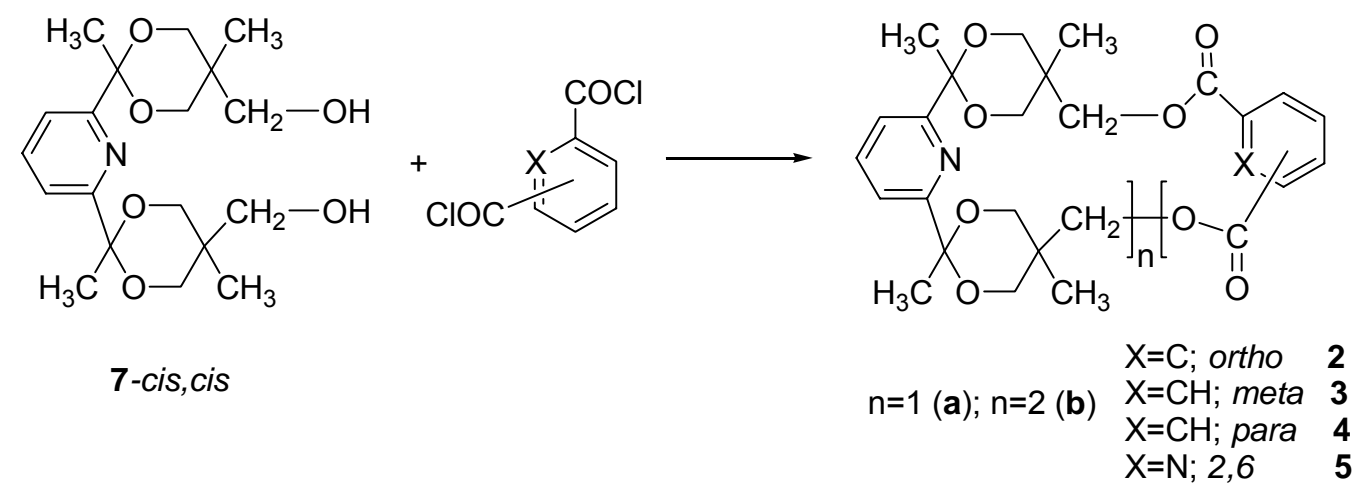

Scheme 1

The complexation reaction of 5a with $\mathrm{CF}_{3} \mathrm{SO}_{3} \mathrm{Ag}$, in $\mathrm{DCM}$ gave the complex 6a $\left[\left(\mathrm{C}_{26} \mathrm{H}_{30} \mathrm{~N}_{2} \mathrm{O}_{8}\right)_{2} \mathrm{Ag}_{4}\right](\mathrm{OTf})_{4} \cdot 4 \mathrm{H}_{2} \mathrm{O}$ in $85 \%$ yield.

The complex $\left[\left(\mathrm{C}_{26} \mathrm{H}_{30} \mathrm{~N}_{2} \mathrm{O}_{8}\right)_{2}(\mathrm{OTf})_{2}\left(\mathrm{H}_{2} \mathrm{O}\right)_{4} \mathrm{Ag}_{4}\right](\mathrm{OTf})_{2} \cdot 2 \mathrm{CHCl}_{3}$ was crystallized from $\mathrm{CHCl}_{3}$-pentane.

(1) Balog, M.; Grosu, I.; Plé, G.; Ramondenc, Y.; Condamine, E.; Varga, R. A. J. Org. Chem., 2004, 69, 13371345

(2) Christensen, C. A.; Batsanov, A. S.; Bryce, M. R.; Howard, J. A. K. J. Org. Chem. 2001, 66, 3313-3320.

(3) Balog, M.; Tötös, S.; Florian, M. C.; Grosu, I.; Plé, G.; Toupet, L.; Ramondenc, Y.; Dincă, N. Heterocyclic Commun. 2004, 10, 139-144 


\section{Experimental determination of the barriers of rotation for compounds 2-5.}

The barriers of rotation were estimated using the temperatures of coalescence and the chemical shifts measured in the frozen structures for the protons of the 5'(5")- $\mathrm{CH}_{2}$ groups and of the equatorial and axial protons of the 1,3-dioxane rings (Table 1). The variable temperature ${ }^{1} \mathrm{H}$ NMR experiments were carried out by the recording of spectra every 10 degrees. The standard deviations were established using the $\Delta G^{\#}$ values calculated at the temperature of observed coalescence $(\mathrm{T}), \mathrm{T}_{\mathrm{c}}+10$ and $\mathrm{T}_{\mathrm{c}}-10$.

The calculations were carried using the equations (1) and (2) ${ }^{4}$

$$
k_{\text {coal. }}=\frac{1}{2} \sqrt{2} \sqrt{\left(\delta_{1}-\delta_{2}\right)^{2}+6 J^{2}}
$$

where :

$\mathrm{k}_{\text {coal. }}$ - rate of site exchange between two equally populated site $\Delta \delta=\delta_{1}-\delta_{2}-$ is the chemical shift difference (in hertz) between the two exchanging nuclei at a temperature well below coalescence.

$J$ - constant coupling of the two nuclei in question

$k_{\text {coal. }}=k\left(k_{B} T / h\right) e^{-\Delta G^{\#} / R T}$

where :

$\mathrm{k}$-the transmission coefficient (usually taken as unity).

$\mathrm{K}_{\mathrm{B}}$-Boltzmann's constant.

$\mathrm{h}-$ Plank's constant.

$\mathrm{T}$-the coalescence temperature

$\Delta \mathrm{G}^{\#}$-the free energy of activation.

Table 1. Free energies of activation calculated from the coalescence temperatures and the chemical shifts of the signals of the protons 5'(5")-CHH, 4'(4')- $\mathrm{H}_{\mathrm{ax}}, 6^{\prime}\left(6^{\prime \prime}\right)-\mathrm{H}_{\mathrm{ax}}, 4^{\prime}\left(4^{\prime \prime}\right)-\mathrm{H}_{\mathrm{eq}}$ and 6'(6") $-\mathrm{H}_{\mathrm{eq}}$ measured in the low temperature ${ }^{1} \mathrm{H}$ NMR spectra $\left(\mathrm{CDCl}_{3}, 400 \mathrm{MHz}\right)$ for compounds $\mathbf{2}-\mathbf{5}$.

\begin{tabular}{|c|c|c|c|c|c|c|c|c|c|c|}
\hline & \multicolumn{3}{|c|}{$\mathbf{T}(\mathrm{K})$} & \multicolumn{3}{|c|}{$\Delta \delta(\mathrm{Hz})$} & \multicolumn{3}{|c|}{$\Delta G^{\#}$ (kcal / mol) using } & \multirow{3}{*}{$\begin{array}{c}\text { Mean } \\
\Delta \mathbf{G}^{\#} \text { (kcal / } \\
\text { mol) values }\end{array}$} \\
\hline & \multirow{2}{*}{$\begin{array}{l}5^{\prime}\left(5^{\prime \prime}\right) \\
\text { СНH }\end{array}$} & \multicolumn{2}{|c|}{ 4'(4")-6'(6") } & \multirow{2}{*}{$\begin{array}{l}5^{\prime}\left(5^{\prime \prime}\right) \\
\text { СНH }\end{array}$} & \multicolumn{2}{|c|}{ 4'(4")-6'(6") } & \multirow{2}{*}{$\begin{array}{l}5^{\prime}\left(5^{\prime \prime}\right) \\
\text { СНH }\end{array}$} & \multicolumn{2}{|c|}{ 4'(4")-6'(6") } & \\
\hline & & $\mathbf{H}_{\mathrm{ax}}$ & $\mathbf{H}_{\mathrm{eq}}$ & & $\mathbf{H}_{\mathrm{ax}}$ & $\mathbf{H}_{\mathrm{eq}}$ & & $\mathbf{H}_{\mathrm{ax}}$ & $\mathbf{H}_{\mathrm{eq}}$ & \\
\hline $2 a^{*}$ & 245 & 245 & 235 & 218.1 & 108.8 & 40.16 & 11.2 & 11.5 & 11.5 & 11.4 \\
\hline $3 \mathbf{a}$ & 323 & 323 & 323 & 501.96 & 138.94 & 144.7 & 14.1 & 15.3 & 15.3 & 14.9 \\
\hline $\mathbf{4 a}^{\#, \$, \dagger}$ & $>393$ & $>393$ & $>393$ & 138 & 24 & 111 & $>18.7$ & $>20.1$ & $>18.9$ & - \\
\hline $4 b^{*, f}$ & 225 & - & - & 486.65 & - & - & 9.2 & - & - & 9.2 \\
\hline $5 \mathbf{a}$ & 303 & 303 & 303 & 503.92 & 138.44 & 55.22 & 14.0 & 14.3 & 14.6 & 14.3 \\
\hline
\end{tabular}

$\mathrm{CD}_{2} \mathrm{Cl}_{2} ;{ }^{\sharp} \mathrm{DMSO}-d_{6} ;{ }^{\circledR} 300 \mathrm{MHz}$;

${ }^{\dagger}$ The coalescence was not obtained till $393 \mathrm{~K}$

${ }^{£}$ Only the signals for 5'(5”)-CHH protons could be assigned in the low temperature spectrum

(4) a) Kurland, R., J. ; Rubin, M., B.; Wise, W. B. J. Phys. Chem, 1964, 40, 2426-2427; b) Eliel, E. L.; Wilen, S. H. Stereochemistry of Organic Compounds John Wiley and Sons: Chichester, UK, 1994, pp 504; c) Eliel, E. L.; Wilen, S. H.; Doyle, M. P. BasicOrganic Stereochemistry, Wiley Interscience, 2001, New York, pp 327 


\section{Molecular modeling for compounds $2 \mathbf{2}-5 \mathbf{a}$}

\subsection{Geometry optimizations}

Full geometry optimizations of $\mathbf{2 a}-\mathbf{5 a}$ have been done at the B3lyp/6-31G(d) level ${ }^{5}$ of theory by using Gaussian98 suite of programs.

The modulation results indicate the syn structure to be the most stable conformer for compounds 2a, 3a and 5a. (Figure 1, Table 2).

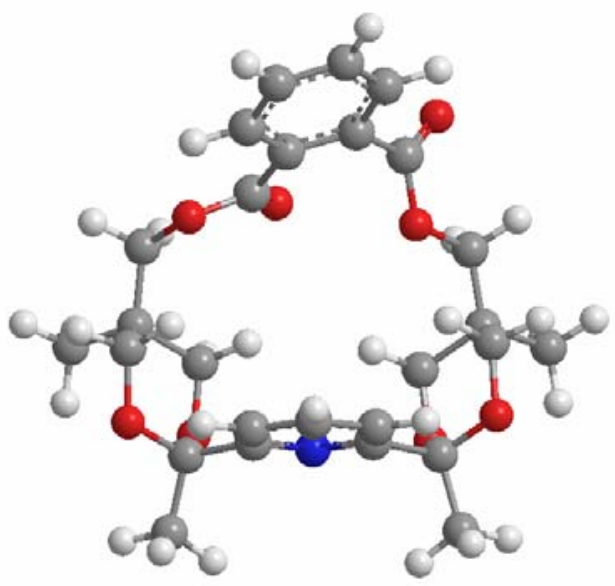

2a-syn

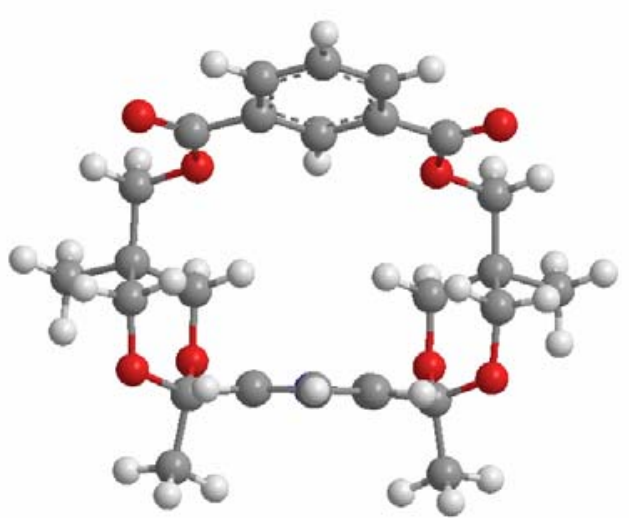

3a-syn

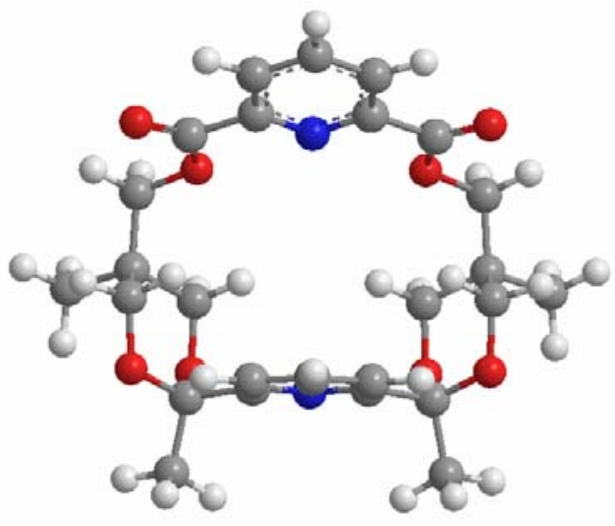

5a-syn

Figure 1. B3LYP/6-31G(d) calculated structures of syn conformers for $\mathbf{2 a}, \mathbf{3 a}$ and $\mathbf{5 a}$

(5) Becke, A.D.; J.Chem.Phys., 1993, 98, 5648-5652. 
In the case of $\mathbf{4 a}$, two conformations with small difference of their relative energies were found to be stable (Figure 2, Table 2).

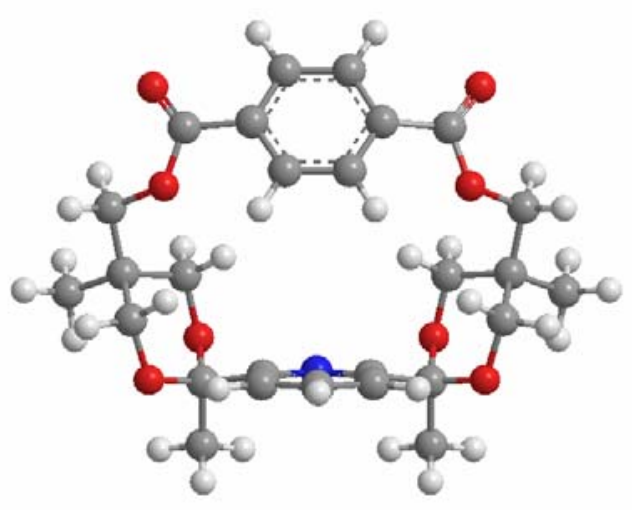

$4 a(I)$

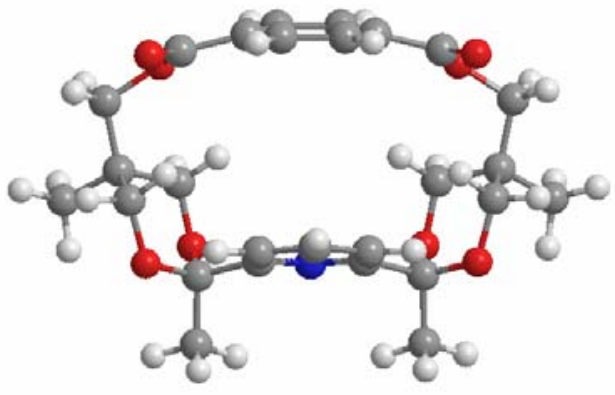

4a (II)

Figure 2. B3LYP/6-31G(d) calculated structures for 4a

Table 2 B3LYP/6-31G(d) total and relative energies for $\mathbf{2 a}-\mathbf{5 a}$.

\begin{tabular}{cccc}
\hline Compound & $\begin{array}{c}\text { Total energies } \\
\text { (a.u.) }\end{array}$ & $\begin{array}{c}\text { Relative } \\
\text { energy } \\
\text { (kcal/mol) }\end{array}$ & $\begin{array}{c}\text { Differences of relative } \\
\text { energies (syn-anti; } \\
\text { kcal/mol) }\end{array}$ \\
\hline $\mathbf{2 a}-$ syn & -1704.090588 & 8.41 & 1.71 \\
$\mathbf{2 a}-$ anti & -1704.087859 & 10.13 & \\
$\mathbf{3 a}-$ syn & $-\mathbf{1 7 0 4 . 1 0 3 9 9 5}$ & $\mathbf{0}$ & 8.37 \\
$\mathbf{3 a}-$ anti & -1704.090656 & 8.37 & \\
$\mathbf{4 a}(\mathbf{I})$ & -1704.086569 & 10.93 & 1.35 \\
$\mathbf{4 a}(\mathbf{I I})$ & -1704.084414 & 12.29 & 7.72 \\
$\mathbf{5 a}-$ syn & -1720.133218 & - & \\
$\mathbf{5 a}-$ anti & -1720.120911 & - & \\
\hline
\end{tabular}




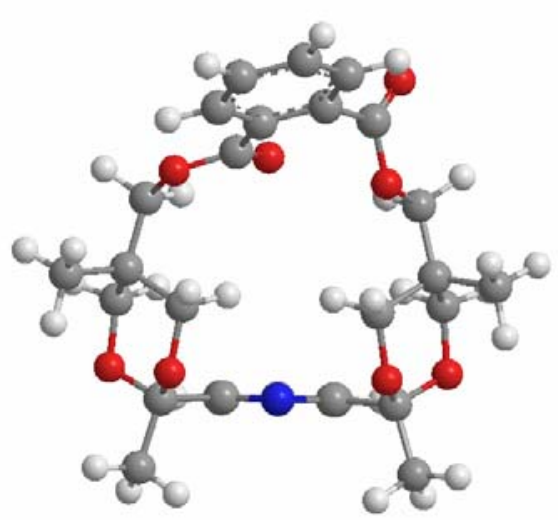

$\mathbf{2 a}-$ anti

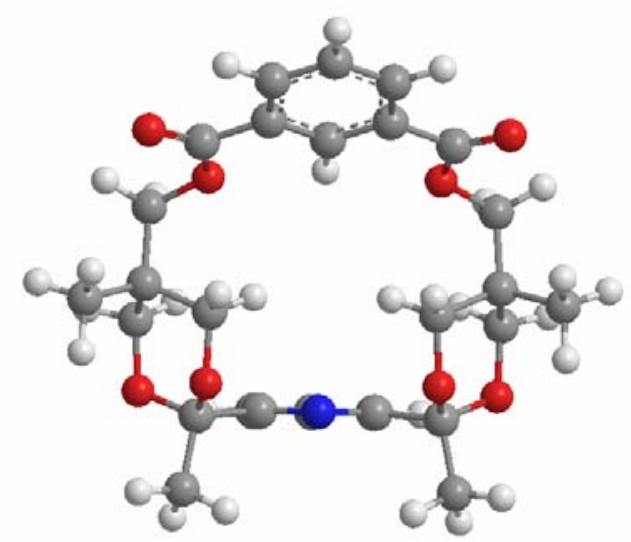

$\mathbf{3 a}-$ anti

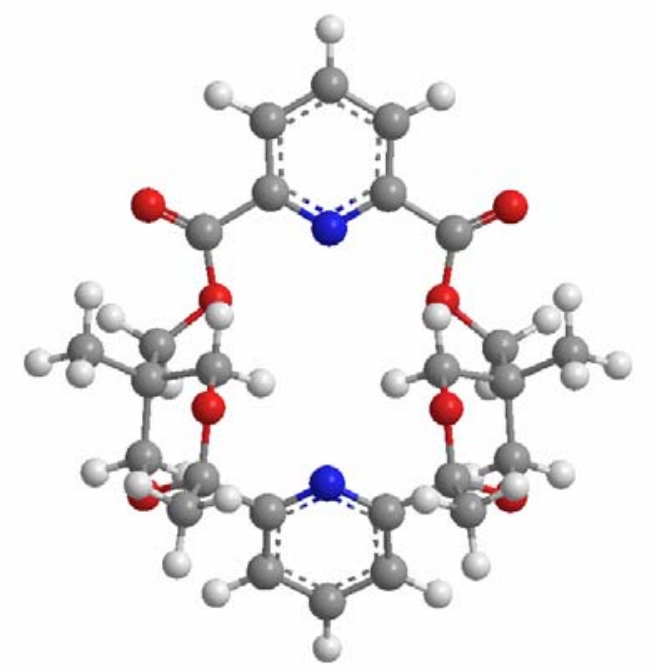

$\mathbf{5 a}-$ anti

Figure 3. B3LYP/6-31G(d) calculated structures of the anti isomers for $\mathbf{2 a}, \mathbf{3 a}$ and $\mathbf{5 a}$.

The syn structures are considerably more stable than the anti structures for 3a and 5a (Table 1). The difference between the relative energies of the syn and anti isomers of $\mathbf{2 a}$ is lower $(1.34 \mathrm{kcal} / \mathrm{mol}$, Table 2$)$. These data are in agreement with low temperature ${ }^{1} \mathrm{H}$ - NMR spectra for 2a, 3a and 5a. As revealed in the paper (Figure 1, paper), the ${ }^{1} \mathrm{H}-\mathrm{NMR}$ spectra of 3a and 5a at $253 \mathrm{~K}\left(\mathrm{CDCl}_{3}\right)$ show a single set of signals, corresponding to the syn isomer. The ${ }^{1} \mathrm{H}-\mathrm{NMR}$ spectra of $\mathbf{2 a}$ at $185 \mathrm{~K}\left(\mathrm{CD}_{2} \mathrm{Cl}_{2}\right.$, Figure 4$)$ show two sets of signals corresponding of syn and anti isomers in the ratio of syn:anti $=3 / 1$. 


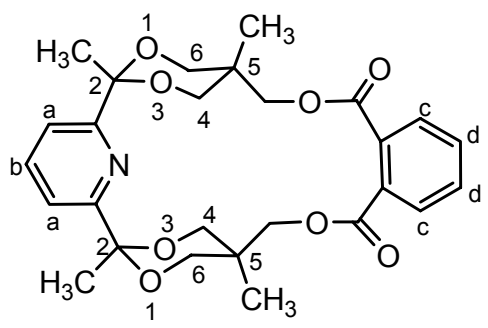

(A)

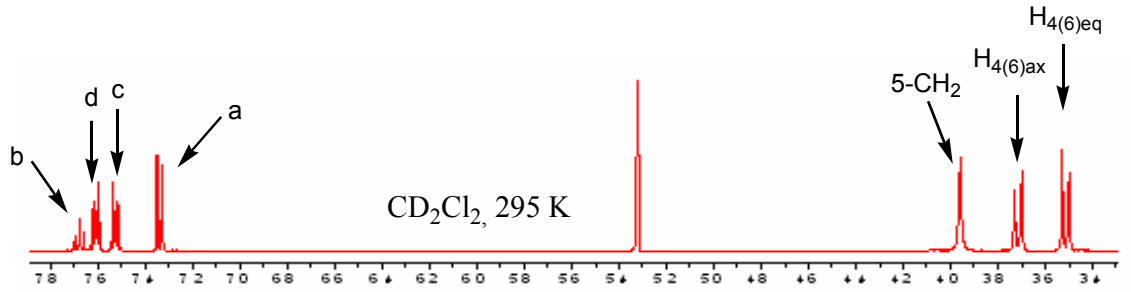

$\mathrm{CD}_{2} \mathrm{Cl}_{2}, 225 \mathrm{~K}$

(B)
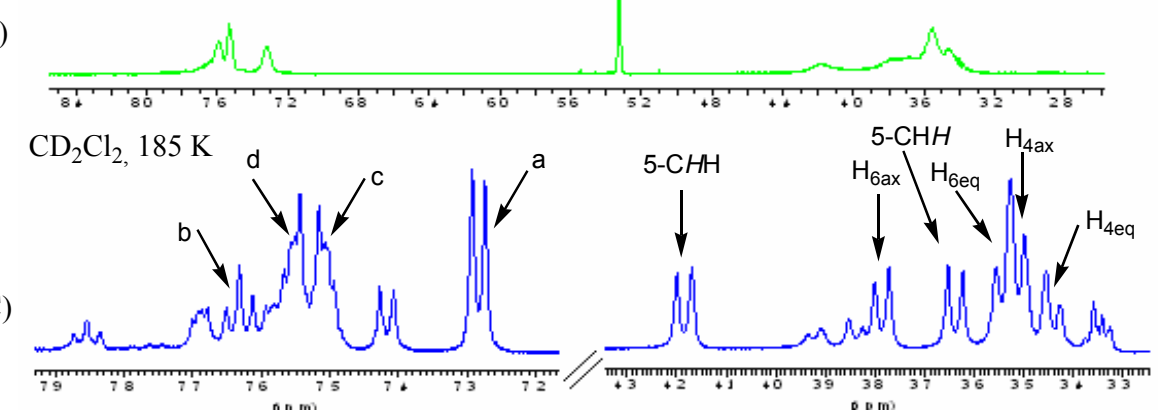

Figure $4{ }^{1} \mathrm{H}$ - NMR spectra of $\mathbf{2 a}$ at $295 \mathrm{~K}$ (A), $225 \mathrm{~K}$ (B) and $185 \mathrm{~K}$ (C). The assigned peaks in the spectrum at $185 \mathrm{~K}$ pertain to syn isomer.

\subsection{Barriers of rotation}

The rotation barriers have been determined from the energy profile calculations at the PM3 level by using Spartan $04{ }^{6}$. SCRF Energy calculations have been then carried out within the $\mathrm{CPCM}_{\text {model }}{ }^{7,8}$ implemented in Gaussian $98^{9}$

(6) Spartan'04, Wavefunction Inc., 18401 Von Karman Avenue, Suite 370' Irvine, CA 92612

(7) Klamt, A.; Schüürmann, G. J. Chem. Soc. Perkin Trans. 2 1993, 799-806.

(8) V. Barone and M. Cossi, J. Phys. Chem. A, 1998, 102, 1995-2001 .

(9) Gaussian 98, Revision A.11.3, Frisch, M. J.; Trucks, G. W.; Schlegel, H. B.; Scuseria, G. E.; Robb, M. A.; Cheeseman, J. R; Zakrzewsk,i V. G.;. Montgomery, J. A Jr.;.. Stratmann, R. E; Burant, J. C.; Dapprich, S.; Millam, J. M.; Daniels, A. D.; Kudin, K. N. ; Strain, M. C.; Farkas, O.; Tomasi, J.; Barone, V.; Cossi, M.; Cammi, R.; Mennucci, B. ; Pomelli, C.; Adamo, C.; Clifford, S.; Ochterski, J.; Petersson, G. A.; Ayala, P. Y.; Cui, Q.; Morokuma, K. ; Rega, N.; Salvador, P. ; Dannenberg, J. J.; Malick, D. K. ; Rabuck, A. D.; Raghavachari, K.; Foresman, J. B.; Cioslowski, J.; Ortiz, J. V. ; Baboul, A. G. ; Stefanov, B. B. ; Liu, G.; Liashenko, A.; Piskorz ,P.; Komaromi, I.; Gomperts, R.; Martin, R. L. ; Fox, D. J; Keith, T.; Al-Laham, M. A.; Peng, C. Y.; Nanayakkara, A.; Challacombe, M.; Gill, P. M. W. ; Johnson, B.; Chen, W. ; Wong, M. W. ; Andres, J. L.; Gonzalez, C.; Head-Gordon, M.; Replogle, E. S.; and Pople, J. A. Gaussian, Inc., Pittsburgh PA, 2002. 
The inward rotation of the pyridine ring A "inside" of macrocycle, involving the conformer II, (Scheme 1, in the paper) in $\mathbf{2 a}-\mathbf{5 a}$ is energetically prohibitive [e.g. maximum of energy for 2a was obtained for a conformation with interring angle between the aromatic rings of $\left.20.14^{\circ}\right)$.

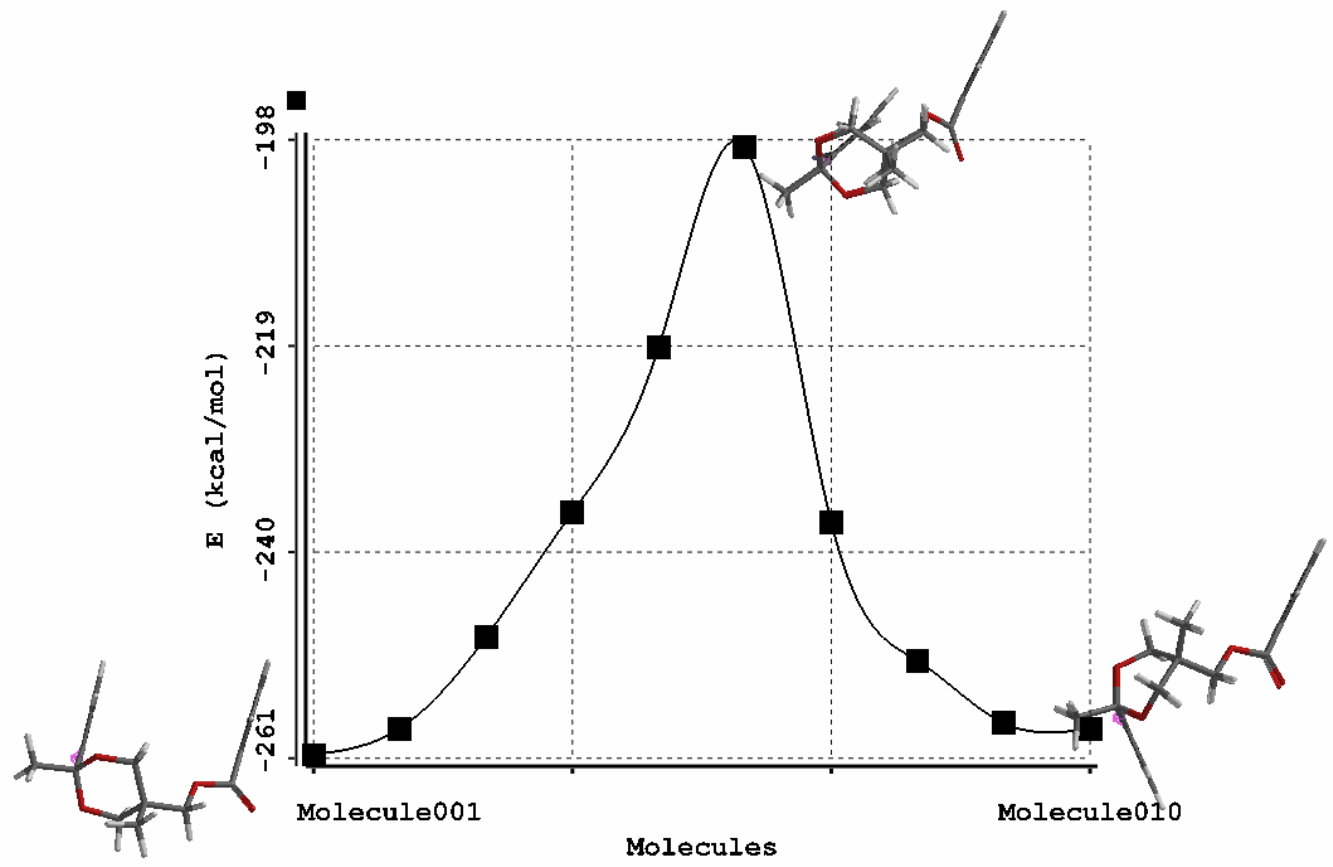

Figure 5. PM3 calculated energy profile for the inward rotation of the pyridine ring in $\mathbf{2 a}$

The rotation barriers of the ring A, involving the conformer III with the nitrogen atom “inside" the macrocycle (Ia $\leftrightarrows$ III $\leftrightarrows$ Ib) (Scheme 1, paper) are shown in table 3, figures 6 and 7. 
Table 3. B3LYP/6-31G(d)//PM3 barriers of rotation of the pyridine ring in 2a - 5a

\begin{tabular}{ccc}
\hline Compound & $\begin{array}{c}\text { Total energies } \\
\text { (a.u.) }\end{array}$ & $\begin{array}{c}\text { Relative energies } \\
\text { (kcal/mol) }\end{array}$ \\
\hline 2a-syn & -1704.061189 & 13.48 \\
2a max & -1704.039714 & \\
3a-syn & -1704.082672 & 34.13 \\
3a max & -1704.028282 & \\
4a(I) & -1704.048667 & 43.14 \\
4a max & -1703.979927 & \\
5a-syn & -256.6 & 26.9 \\
5a max & -227.7 & \\
\hline
\end{tabular}

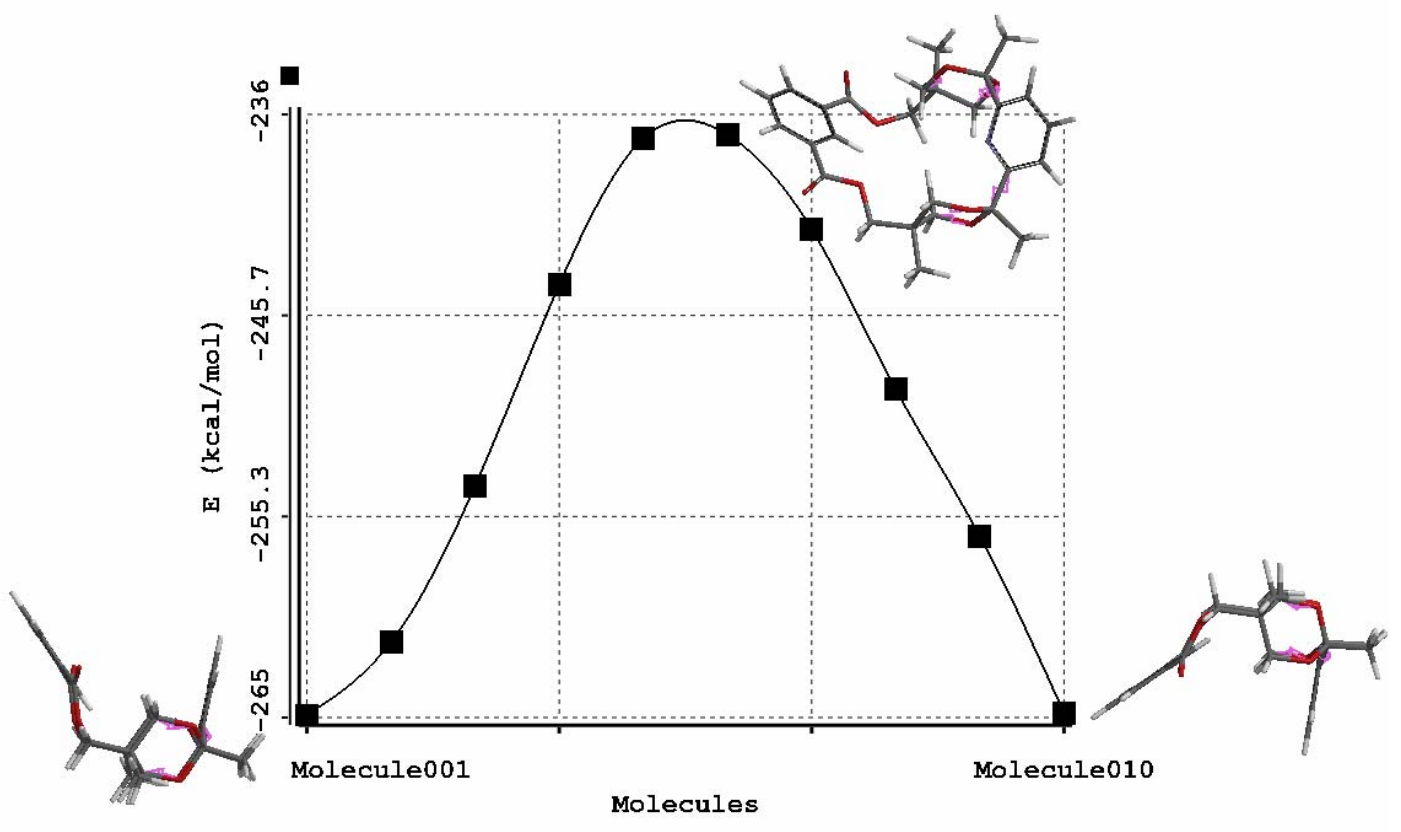

Figure 6. PM3 calculated energy profile for the outward rotation of the pyridine ring in $\mathbf{3 a}$ 


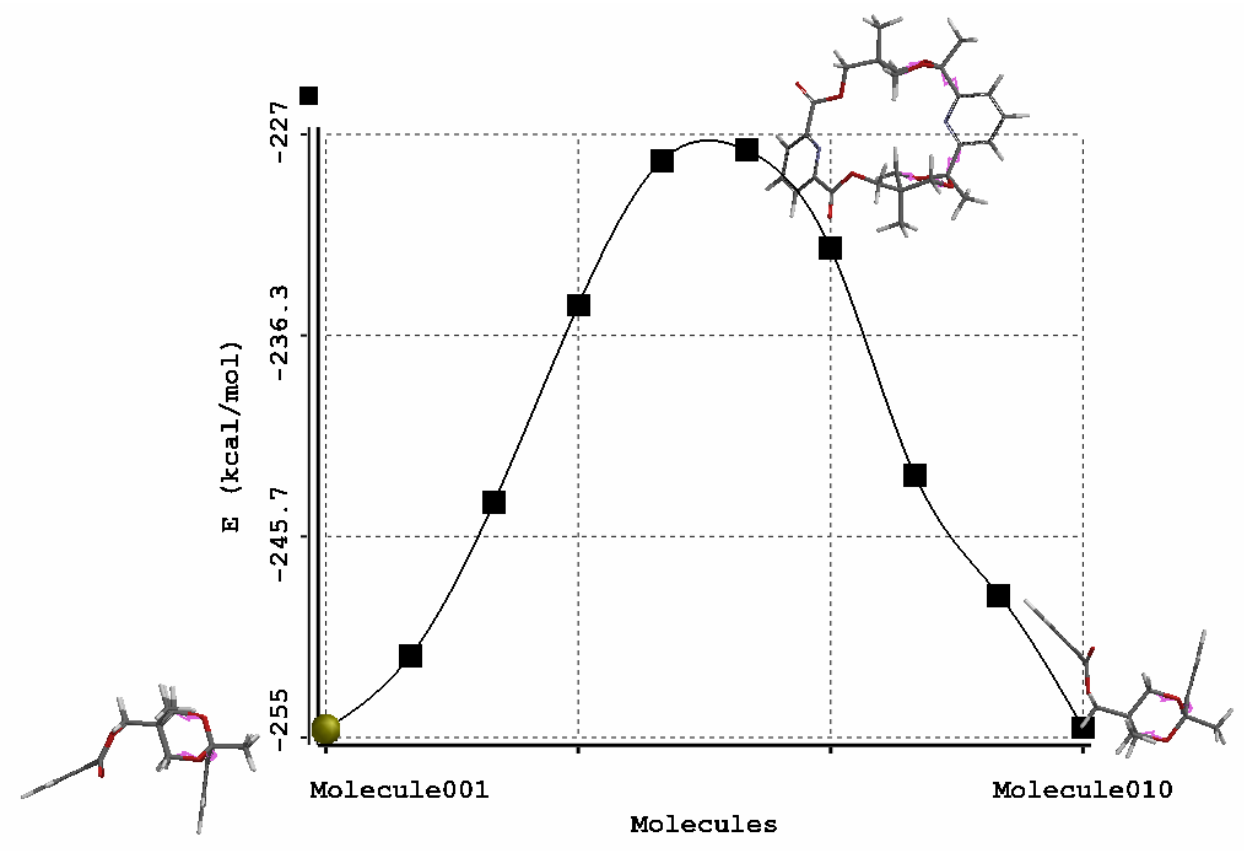

Figure 7. PM3 calculated energy profile for the outward rotation of pyridine A in 5a

\section{X-ray Crystalographic Data}

Table 4. Torsion angles for different conformers of 2a (A-D)

\begin{tabular}{|c|c|c|c|c|}
\hline \multirow{2}{*}{ Structure } & \multicolumn{3}{|c|}{ Torsion angle } & \multirow{2}{*}{$\begin{array}{c}\text { Torsion angle between the } \\
\text { two carbonyl groups }\end{array}$} \\
\cline { 2 - 4 } & $\mathrm{P} 1 / \mathrm{P} 2$ & $\mathrm{P} 1 / \mathrm{P} 3$ & $\mathrm{P} 1 / \mathrm{P} 4$ & 48.44 \\
\hline $\mathbf{2 a}(\mathbf{A})$ & 88.92 & 84.89 & 27.54 & 45.89 \\
\hline $\mathbf{2 a}(\mathbf{B})$ & 89.90 & 83.12 & 32.09 & 45.83 \\
\hline 2a (C) & 89.14 & 80.39 & 28.57 & 47.16 \\
\hline $\mathbf{2 a}(D)$ & 87.81 & 87.72 & 30.81 & \multicolumn{2}{c}{} \\
\hline
\end{tabular}

P1 plane of pyridine ring A (C9 C10 C11 C12 C13 N28)

$\mathrm{P} 2$ plane of (C17 C18 C34)

P3 plane of (C4 C5 C31)

$\mathrm{P} 4$ plane of aromatic ring B (C21 C1 C25 C24 C23 C22) 
Table 5. Selected angles and distances for the complex $6 \mathbf{a}$

\begin{tabular}{|c|c|c|c|}
\hline $\operatorname{Ag}(1)-N(30)$ & 2.313 & $\operatorname{Ag}(2)-N(27)$ & 2.378 \\
\hline $\operatorname{Ag}(1)-\mathrm{O}(3)$ & 2.632 & $\operatorname{Ag}(2)-O(7)$ & 2.654 \\
\hline $\mathrm{Ag}(1)-\mathrm{O}(7)$ & 2.699 & $\operatorname{Ag}(2)-O(46)$ & 2.539 \\
\hline $\mathrm{Ag}(1)-\mathrm{O}(48)$ & 2.235 & $\operatorname{Ag}(2)-O(47)$ & 2.391 \\
\hline $\mathrm{Ag}(1)-\mathrm{O}(19)$ & 2.634 & $\mathrm{Ag}(2)-\mathrm{O}(44)$ & 2.552 \\
\hline $\operatorname{Ag}(1)-\operatorname{Ag}(2)$ & 3.325 & $\operatorname{Ag}(2)-\operatorname{Ag}(28)$ & 2.558 \\
\hline $\mathrm{O}(19)-\mathrm{Ag}(1)-\mathrm{N}(30)$ & 66.46 & $\mathrm{O}(47)-\mathrm{Ag}(2)-\mathrm{O}(46)$ & 79.49 \\
\hline $\mathrm{N}(30)-\operatorname{Ag}(1)-\mathrm{O}(3)$ & 66.63 & $\mathrm{O}(44)-\mathrm{Ag}(2)-\mathrm{O}(46)$ & 84.09 \\
\hline $\mathrm{O}(3)-\mathrm{Ag}(1)-\mathrm{O}(7)$ & 65.27 & $\mathrm{O}(44)-\mathrm{Ag}(2)-\mathrm{O}(28)$ & 76.76 \\
\hline $\mathrm{O}(19)-\mathrm{Ag}(1)-\mathrm{O}(48)$ & 96.18 & $\mathrm{O}(28)-\mathrm{Ag}(2)-\mathrm{O}(7)$ & 103.61 \\
\hline $\mathrm{O}(19)-\mathrm{Ag}(1)-\mathrm{O}(3)$ & 127.91 & $\mathrm{O}(28)-\mathrm{Ag}(2)-\mathrm{O}(47)$ & 131.44 \\
\hline $\mathrm{O}(19)-\mathrm{Ag}(1)-\mathrm{O}(7)$ & 156.05 & $\mathrm{O}(28)-\mathrm{Ag}(2)-\mathrm{O}(46)$ & 140.71 \\
\hline $\mathrm{N}(30)-\mathrm{Ag}(1)-\mathrm{O}(7)$ & 111.07 & $\mathrm{O}(7)-\mathrm{Ag}(2)-\mathrm{O}(46)$ & 102.84 \\
\hline $\mathrm{N}(30)-\operatorname{Ag}(1)-\mathrm{O}(48)$ & 151.41 & $\mathrm{O}(7)-\mathrm{Ag}(2)-\mathrm{O}(44)$ & 85.11 \\
\hline $\mathrm{O}(3)-\mathrm{Ag}(1)-\mathrm{O}(7)$ & 115.9 & $\mathrm{O}(44)-\mathrm{Ag}(2)-\mathrm{O}(7)$ & 148.60 \\
\hline $\mathrm{O}(28)-\mathrm{Ag}(2)-\mathrm{N}(27)$ & 63.10 & $\mathrm{O}(47)-\mathrm{Ag}(2)-\mathrm{N}(27)$ & 146.25 \\
\hline $\mathrm{N}(27)-\operatorname{Ag}(2)-\mathrm{O}(7)$ & 62.02 & $\mathrm{O}(46)-\mathrm{Ag}(2)-\mathrm{N}(27)$ & 106.49 \\
\hline $\mathrm{O}(7)-\mathrm{Ag}(2)-\mathrm{O}(47)$ & 84.23 & $\mathrm{O}(44)-\mathrm{Ag}(2)-\mathrm{N}(27)$ & 128.16 \\
\hline
\end{tabular}

The lattice of the complex $\mathbf{6 a}$ is organized in channels that are filled with molecules of chloroform (Figure 8).

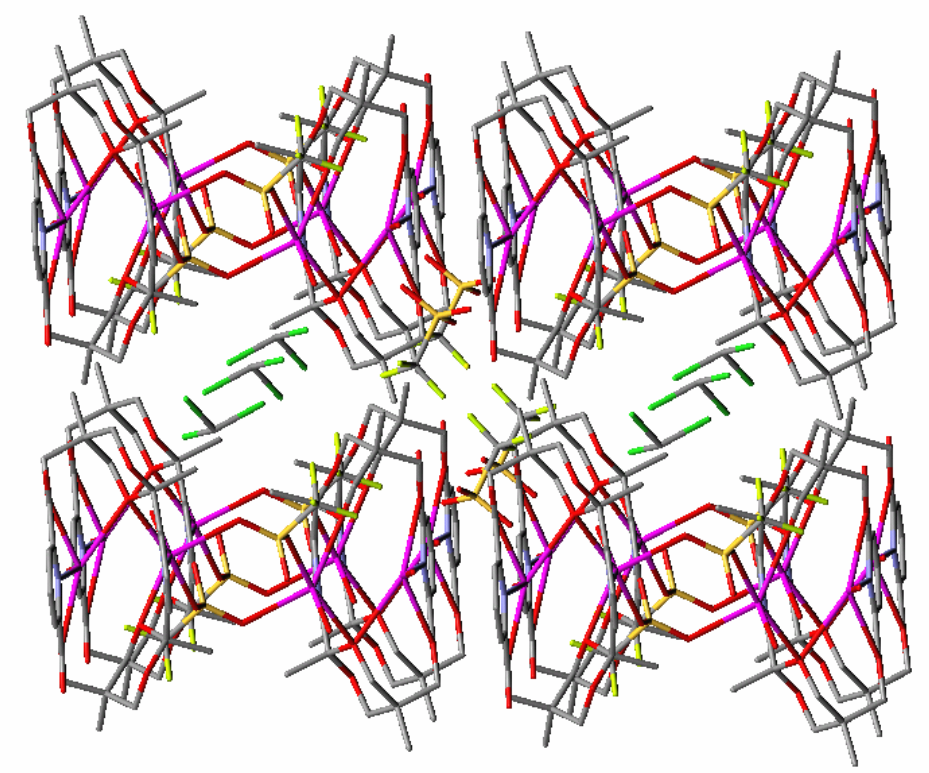

Figure 8. View of the lattice of complex $\mathbf{6 a}$ along a axis. 


\section{Experimental section}

\subsection{General experimental data}

Unless otherwise states, routine ${ }^{1} \mathrm{H}-\mathrm{NMR}(300 \mathrm{MHz})$ and ${ }^{13} \mathrm{C}-\mathrm{NMR}(75 \mathrm{MHz})$ spectra, COSY, HMQC, $\mathrm{HMBC}$ were recorded at $r t$ in $\mathrm{CDCl}_{3}$ on a Bruker $300 \mathrm{MHz}$ spectrometer, using the solvent line as reference. Variable temperature NMR spectra and low temperature ROESY and NOESY were recorded on Bruker ARX 400 MHz and Bruker Avance DMX 500 spectrometers respectively, in $\mathrm{CDCl}_{3}, \mathrm{CD}_{2} \mathrm{Cl}_{2}$ or DMSO- $d_{6}$. Melting points were measured with a Kleinfeld melting point apparatus and are uncorrected. Microanalyses $(\mathrm{C}, \mathrm{H}, \mathrm{N})$ agreed (inside $+/-0.4 \%$ ) with calculated data. Mass spectra were recorded on an ATI Unicam Automass, a Micromass TofSpc E or a JEOL- AX 500 spectrometers, respectively.

Thin-layer chromatography (TLC) was conducted on silica gel $60 \mathrm{~F}_{254}$ TLC plates purchased from Merck. Preparative column (flash) chromatography was preformed using PharmPrep 60 CC (40-63 $\mu \mathrm{m})$ silica gel purchased from Merck.

The experimental conditions for the X-ray structure determination of compound 2a, 5a, 6a and details of the refinements are given in the pages below.

The structural data were deposited at the Cambridge Crystallographic Data Center, deposition numbers CCDC 273418 (for 2a), CCDC 284739 (for 5a) and CCDC 292379 (for 6a)

Chemicals were purchased from Aldrich or Acros and were used without further purification.

\subsection{General procedure for the synthesis of diol 7}

1,1,1-Tris(hydroxymethyl)ethane (6.10 mmol, 1 equiv.), 2,6-diacetyl-pyridine (6.10 mmol, 1 equiv.) and p-toluenesulfonic acid (7.97 mmol, 1.3 equiv.) were dissolved in $40 \mathrm{~mL}$ benzene. The mixture was refluxed for $72 \mathrm{~h}$, and $\mathrm{H}_{2} \mathrm{O}$ was removed with a Dean-Stark trap. When 80 $\%$ of the water was separated the mixture was cooled to $r t$, and the catalyst was neutralized (stirring $0.5 \mathrm{~h}$ with $1.3 \mathrm{~g}$ sodium carbonate). The benzene was removed and the resulted solid was solved in dichloromethane $(40 \mathrm{~mL})$. The solution was stirred for $0.5 \mathrm{~h}$, washed twice with $20 \mathrm{~mL}$ water and dried with $\mathrm{MgSO}_{4}$. Filtration and evaporation of the solvent lead to a mixture of three diastereoisomers cis-cis, cis-trans and trans-trans in a ratio of 1.1/1.8/1 (78 $\%$ yields). The mixture was separated over silicagel and eluted with dichloromethane/ethanol/ ammonia (4.7/0.3/0.01) to afford the pure cis-cis isomer 7 in $16 \%$ yields. 
5.3 General procedure for the preparation of the [7.7]cyclophanes 2a-5a and [7.7.7.7]cyclophane $4 \mathrm{~b}$

A solution of phthaloyl dichloride ( $0.66 \mathrm{mmol}, 1.2$ equiv.) in dry THF (20 mL) was added with a high precision push-syringe over a period of $12 \mathrm{~h}$ to a well stirred solution of diol 1 (0.54 mmol, 1 equiv.) in dry $\mathrm{CH}_{3} \mathrm{CN}(120 \mathrm{~mL})$ and dry THF $(30 \mathrm{~mL})$ containing N,N'dimethyl-4-aminopyridine (DMAP, $0.80 \mathrm{mmol}, 1.48$ equiv.). The mixture was stirred at $r t$ and the reaction was monitored by TLC. Solvents were evaporated in vacuo, the residue was triturated with ether $(3 \times 50 \mathrm{~mL})$ and the ether extract was evaporated in vacuo. The residue was chromatographed on silica gel to afford the target macrocyclic cyclophanes.

Observation! In the case of compound 3a and 5a the cyclophanes were precipitated from the crude mixture of reaction. The pure products were collected by suction filtrations and washed several time with THF.

\subsection{General procedure for synthesis of complex 6 a}

To a stirred solution of $\mathrm{CF}_{3} \mathrm{SO}_{3} \mathrm{Ag}(0.03 \mathrm{mmol}, 2$ equiv.) in $2 \mathrm{~mL}$ dichloromethane was added a solution of $5 \mathbf{a}(0.015 \mathrm{mmol}, 1$ equiv.) in $1.5 \mathrm{~mL}$ dichloromethane. The stirring was maintained for another $2 \mathrm{~h}$. Then, the solvent was removed by vacuum distillation and after addition of $5 \mathrm{~mL}$ pentane, the complex was obtained as white crystals. The product was filtrated of and dried in vacuo. (85\%, yield). Single crystals $\left[\left(\mathrm{C}_{26} \mathrm{H}_{30} \mathrm{~N}_{2} \mathrm{O}_{8}\right)_{2}(\mathrm{OTf})_{2}\left(\mathrm{H}_{2} \mathrm{O}\right)_{4} \mathrm{Ag}_{4}\right](\mathrm{OTf})_{2} \cdot 2 \mathrm{CHCl}_{3}$ were obtained by crystallization from a mixture of $\mathrm{CHCl}_{3} /$ pentane. 


\subsection{Compounds characterization}

\section{5,8,14,17-tetramethyl-2,20-dioxo-28-aza-3,7,15,19,27,29-hexaoxa-}

pentacyclo[19.4. $\left.2^{5,8} \cdot 2^{14,17} \cdot 1^{9,13} \cdot 0^{1,21}\right]$ triaconta-1(21),9(28),10,12,22,24-hexaene (2a)

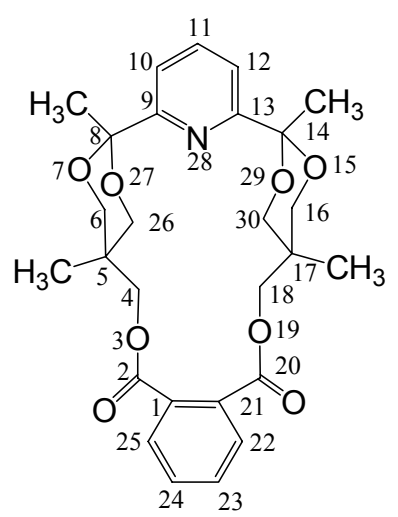

Yield: $31 \%$, white crystals; m.p. $=276-277^{\circ} \mathrm{C}$; purified by flash chromatography (silica, $\mathrm{DCM} / \mathrm{methanol} / \mathrm{ammonia}=4.6 / 0.4 / 0.01) \mathrm{R}_{f}=0.43$.

${ }^{1}$ H NMR (300 MHz, $\left.\mathrm{CDCl}_{3}\right): \delta 1.36\left(\mathrm{~s}, 6 \mathrm{H}, \mathrm{CH}_{3 \mathrm{ax}}-5, \mathrm{CH}_{3 \mathrm{ax}}-17\right), 1.56\left(\mathrm{~s}, 6 \mathrm{H}, \mathrm{CH}_{3 \mathrm{eq}}-8, \mathrm{CH}_{3 \mathrm{eq}}{ }^{-}\right.$ 14), 3.54 (d, $\left.4 \mathrm{H}, \mathrm{H}_{\mathrm{eq}}-6, \mathrm{H}_{\mathrm{eq}}-16, \mathrm{H}_{\mathrm{eq}}-26, \mathrm{H}_{\mathrm{eq}}-30,{ }^{2} J=11.3 \mathrm{~Hz}\right), 3.73\left(\mathrm{~d}, 4 \mathrm{H}, \mathrm{H}_{\mathrm{ax}}-6, \mathrm{H}_{\mathrm{ax}}-16, \mathrm{H}_{\mathrm{ax}}-\right.$ 26, $\mathrm{H}_{\mathrm{ax}}-30,{ }^{2} \mathrm{~J}=11.3 \mathrm{~Hz}$ ), 4.00 (s, 4H, H-4, H-18), 7.34 (d, 2H, H-10, H-12 ${ }^{3} J=7.9 \mathrm{~Hz}$ ), 7.51 (m, 2H, H-23, H-24), 7.62 (m, 2H, H-22, H-25), 7.66 (t, 1H, H-11, ${ }^{3} J=7.9 \mathrm{~Hz}$ ).

${ }^{13}$ C NMR $\left(75 \mathrm{MHz}, \mathrm{CDCl}_{3}\right): \delta 20.0\left(\mathrm{CH}_{3}-5, \mathrm{CH}_{3}-17\right), 29.8\left(\mathrm{CH}_{3}-8, \mathrm{CH}_{3}-14\right), 34.1(\mathrm{C}-5, \mathrm{C}-$ 17), 67.9 (C-6, C-16, C-26, C-30), 69.2 (C-4, C-18), 100.5 (C-8, C-14), 120.8 (C-10, C-12), 129.1 (C-23, C-24), 131.4 (C-1, C21), 131.6 (C-22, C-25), 137.9 (C-11), 160.0 (C-9, C-13), 167.2 (C-2, C-20).

MS (EI, 70 eV) (rel. int., \%) m/z: 498 (M+3), 468 (1), 426 (1), 369 (4), 293 (4), 248 (3), 210 (3), 174 (24), 149 (21), 105 (34), 55 (100).

Calculated for $\mathrm{C}_{27} \mathrm{H}_{31} \mathrm{NO}_{8}$ : C 65.18, H 6.28, N 2.82, found C 65.11, H 6.06, N 2.98 


\section{5,8,14,17-tetramethyl-2,20-dioxo-27-aza-3,7,15,19,26,28-hexaoxa-}

pentacyclo[19.3.2 $\left.2^{5,8} \cdot 2^{14,17} \cdot 1^{9,13} \cdot 1^{1,21}\right]$ triaconta-1(30),9(27),10,12, 21,23-hexaene (3a).

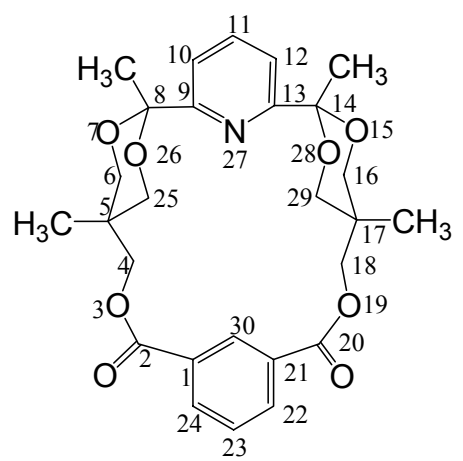

Yield: $50 \%$, white crystals; m.p. $>360{ }^{\circ} \mathrm{C} ;{ }^{1} \mathbf{H}$ NMR (400 MHz, $\mathrm{CDCl}_{3}, 253 \mathrm{~K}$ ): $\delta 1.37$ (s, $\left.6 \mathrm{H}, \mathrm{CH}_{3 \mathrm{ax}}-5, \mathrm{CH}_{3 \mathrm{ax}}-17\right), 1.52$ (s, 6H, $\left.\mathrm{CH}_{3 \mathrm{eq}}-8, \mathrm{CH}_{3 \mathrm{eq}}-14\right), 3.51\left(\mathrm{~d}, 2 \mathrm{H}, \mathrm{CHH}-4, \mathrm{CHH}-18,{ }^{2} \mathrm{~J}=\right.$ $12.0 \mathrm{~Hz}), 3.61\left(\mathrm{dd}, 2 \mathrm{H}, \mathrm{H}_{\mathrm{eq}}-6, \mathrm{H}_{\mathrm{eq}}-16,{ }^{2} \mathrm{~J}=11.2 \mathrm{~Hz},{ }^{4} \mathrm{~J}=2.7 \mathrm{~Hz}\right), 3.77\left(\mathrm{dd}, 2 \mathrm{H}, \mathrm{H}_{\mathrm{eq}}-25, \mathrm{H}_{\mathrm{eq}}{ }^{-}\right.$ $\left.29,{ }^{2} J=11.3 \mathrm{~Hz},{ }^{4} J=2.7 \mathrm{~Hz}\right), 3.78\left(\mathrm{~d}, 2 \mathrm{H}, \mathrm{H}_{\mathrm{ax}}-6, \mathrm{H}_{\mathrm{ax}}-16^{2} \mathrm{~J}=11.2 \mathrm{~Hz}\right), 3.97\left(\mathrm{~d}, 2 \mathrm{H}, \mathrm{H}_{\mathrm{ax}}-25\right.$, $\left.\mathrm{H}_{\mathrm{ax}}-29,{ }^{2} \mathrm{~J}=11.3 \mathrm{~Hz}\right), 4.86\left(\mathrm{~d}, 2 \mathrm{H}, \mathrm{CHH}-4, \mathrm{CH} H-18^{2} \mathrm{~J}=12.0 \mathrm{~Hz}\right), 7.32$ (d, 2H, H-10, H-12, $\left.{ }^{3} J=7.7 \mathrm{~Hz}\right), 7.52\left(\mathrm{t}, 1 \mathrm{H}, \mathrm{H}-11,{ }^{3} J=7.7 \mathrm{~Hz}\right), 7.54\left(\mathrm{t}, 1 \mathrm{H}, \mathrm{H}-23,{ }^{3} J=7.7 \mathrm{~Hz}\right), 7.96(\mathrm{t}, 1 \mathrm{H}, \mathrm{H}-$ $\left.30,{ }^{4} \mathrm{~J}=1.7 \mathrm{~Hz}\right), 8.18\left(\mathrm{dd}, 2 \mathrm{H}, \mathrm{H}-22, \mathrm{H}-24,{ }^{3} \mathrm{~J}=7.7 \mathrm{~Hz},{ }^{4} \mathrm{~J}=1.7 \mathrm{~Hz}\right)$.

${ }^{13}$ C NMR (75 MHz, CDCl $): \delta 19.2\left(\mathrm{CH}_{3}-5, \mathrm{CH}_{3}-17\right), 30.7\left(\mathrm{CH}_{3}-8, \mathrm{CH}_{3}-14\right), 34.9(\mathrm{C}-5, \mathrm{C}-$ 17), 66.8 (C-4, C-18), 67.7 (C-6, C-16, C-25, C-29), 100.6 (C-8, C-14), 120.9 (C-10, C-12), 128.9 (C-23), 129.2 (C-30), 129.9 (C-1, C21), 134.8 (C-22, C-24), 137.6 (C-11), 160.0 (C9, C-13), 164.9 (C-2, C-20).

MS (IC positive, $200 \mathrm{eV}$, gaze: isobutane) (rel int, \%) m/z: $498\left(\mathrm{MH}^{+}, 100\right), 540\left(\mathrm{MC}_{3} \mathrm{H}_{7}^{+}, 1\right)$.

(EI, 70 eV) (rel. int., \%) m/z: 497 (M+27), 468 (13), 426 (25), 369 (19), 305 (2), 275 (6), 248 (30), 203 (39), 149 (61), 104 (70), 55 (100).

Calculated for $\mathrm{C}_{27} \mathrm{H}_{31} \mathrm{NO}_{8}$ : C 65.18, H 6.28, N 2.82, found C 65.33, H 6.47, N 2.70 


\section{5,8,14,17-tetramethyl-2,20-dioxo-26-aza-3,7,15,19,25,27-hexaoxa-pentacyclo-}

$\left[19.2 .2^{5,8} \cdot 2^{14,17} \cdot 1^{9,13}\right]$ triaconta-1(30),9(26),10,12,21(29),22-hexaene (4a)

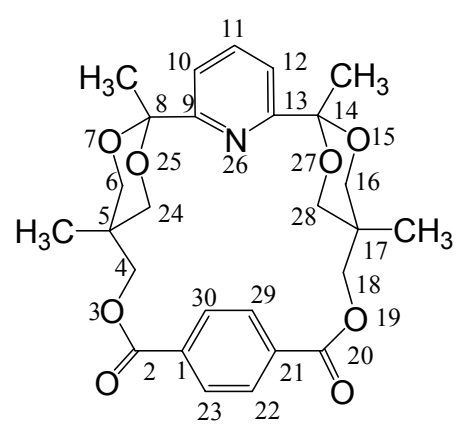

Yield: $27 \%$, white crystals; m.p. $156-157{ }^{\circ} \mathrm{C}$; Purified by flash chromatography (silica, $\mathrm{DCM} /$ methanol/ammonia $=4.7 / 0.3 / 0.01) \mathrm{R}_{f}=0.56$.

${ }^{1}$ H NMR (300 MHz, $\mathrm{CDCl}_{3}$ ): $\delta 1.13\left(\mathrm{~s}, 6 \mathrm{H}, \mathrm{CH}_{3 \mathrm{ax}}-5, \mathrm{CH}_{3 \mathrm{ax}}-17\right), 1.62\left(\mathrm{~s}, 6 \mathrm{H}, \mathrm{CH}_{3 \mathrm{eq}}-8, \mathrm{CH}_{3 \mathrm{eq}}{ }^{-}\right.$ 14), 3.58 (d, 2H, CHH-4, CHH-18, $\left.{ }^{2} \mathrm{~J}=10.9 \mathrm{~Hz}\right), 3.79$ (dd, $2 \mathrm{H}, \mathrm{H}_{\mathrm{eq}}-6, \mathrm{H}_{\mathrm{eq}}-16,{ }^{2} J=12.0 \mathrm{~Hz}$, ${ }^{4} J=0.8 \mathrm{~Hz}$ ), $3.97\left(\mathrm{~d}, 2 \mathrm{H}, \mathrm{H}_{\mathrm{ax}}-6, \mathrm{H}_{\mathrm{ax}}-16^{2} \mathrm{~J}=12.0 \mathrm{~Hz}\right), 4.04\left(\mathrm{~d}, 2 \mathrm{H}, \mathrm{CH}-4, \mathrm{CH}-18{ }^{2} J=\right.$ $10.9 \mathrm{~Hz}), 4.05$ (d, 2H, $\left.\mathrm{H}_{\mathrm{ax}}-24, \mathrm{H}_{\mathrm{ax}}-28^{2} J=11.3 \mathrm{~Hz}\right), 4.16\left(\mathrm{dd}, 2 \mathrm{H}, \mathrm{H}_{\mathrm{eq}}-24, \mathrm{H}_{\mathrm{eq}}-28,{ }^{2} J=11.3\right.$ $\left.\mathrm{Hz},{ }^{4} \mathrm{~J}=0.8 \mathrm{~Hz}\right), 7.38$ (d, 2H, H-10, H-12, $\left.{ }^{3} \mathrm{~J}=7.9 \mathrm{~Hz}\right), 7.61$ (t, $\left.1 \mathrm{H}, \mathrm{H}-11{ }^{3} \mathrm{~J}=7.9 \mathrm{~Hz}\right), 7.94$ (s, 4H, H-22, H-23, H-29, H-30).

${ }^{13}$ C NMR (75 MHz, CDCl $)$ : $\delta 20.0\left(\mathrm{CH}_{3}-5, \mathrm{CH}_{3}-17\right), 22.5\left(\mathrm{CH}_{3}-8, \mathrm{CH}_{3}-14\right), 38.1(\mathrm{C}-5, \mathrm{C}-$ 17), 66.9 (C-4, C-18), 67.6 (C-6, C-16), 68.8 (C-24, C-28), 101.5 (C-8, C-14), 117.9 (C-10, C-12), 130.3 (C-22, C-23, C-29, C-30), 133.6 (C-1, C-21), 137.3 (C-11), 160.9 (C-9, C-13), 165.7 (C-2, C-20).

MS (EI, 70 eV) (rel. int., \%) m/z: 497 (M+47), 419 (16), 380 (12), 248 (11), 203 (23), 149 (59), 104 (100), 55 (79).

Calculated for $\mathrm{C}_{27} \mathrm{H}_{31} \mathrm{NO}_{8}$ : C 65.18, H 6.28, N 2.82, found C 65.09, H 6.36, N 2.93 


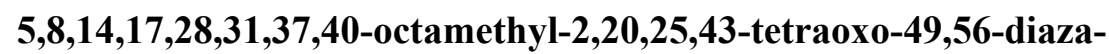

\section{$3,7,15,19,26,30,38,42,48,50,55,57$-dodecaoxa-}

nonacyclo[42.2. $\left.2^{5,8} \cdot 2^{14,17} \cdot 2^{21,24} \cdot 2^{28,31} \cdot 2^{37,40} \cdot 1^{9,13} \cdot 1^{32,36}\right]$ hexaconta-

1(60),9(49),10,12,21(52),22,24(53),32(56),33,35,44(59),45-dodecanene (4b)

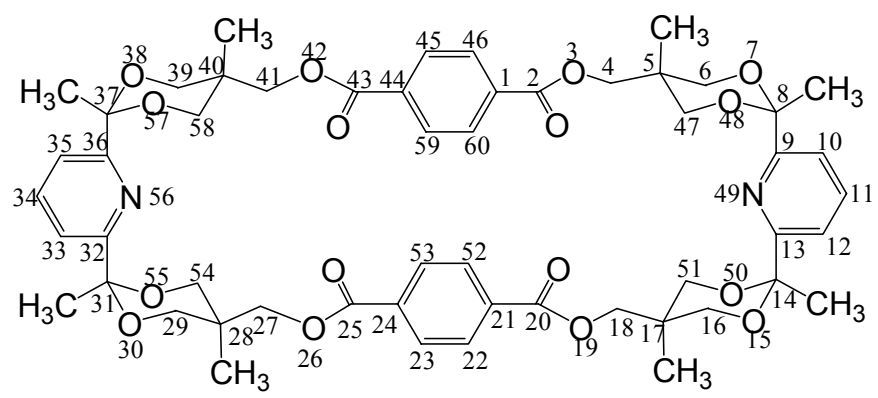

Yield: $7 \%$, white crystals; m.p. $>350{ }^{\circ} \mathrm{C}$ (with decomposition); Purified by flash chromatography (silica, DCM/methanol/ammonia $=4.7 / 0.3 / 0.01) \mathrm{R}_{f}=0.44$.

${ }^{1}$ H NMR (500 MHz, $\left.\mathrm{CD}_{2} \mathrm{Cl}_{2}\right): \delta 1.33\left(\mathrm{~s}, 12 \mathrm{H}, \mathrm{CH}_{3 \mathrm{ax}}-5, \mathrm{CH}_{3 \mathrm{ax}}-17, \mathrm{CH}_{3 \mathrm{ax}}-28, \mathrm{CH}_{3 \mathrm{ax}}-40\right), 1.59$ (s, 12H, $\mathrm{CH}_{3 \mathrm{eq}}-8, \mathrm{CH}_{3 \mathrm{eq}}-14, \mathrm{CH}_{3 \mathrm{eq}}-31, \mathrm{CH}_{3 \mathrm{eq}}-37$ ), 3.64 (s, 8H, H-4, H-18, H-27, H-41), 3.67 (d, $8 \mathrm{H}, \mathrm{H}_{\mathrm{eq}}-6, \mathrm{H}_{\mathrm{eq}}-16, \mathrm{H}_{\mathrm{eq}}-29, \mathrm{H}_{\mathrm{eq}}-39, \mathrm{H}_{\mathrm{eq}}-47, \mathrm{H}_{\mathrm{eq}}-51, \mathrm{H}_{\mathrm{eq}}-54, \mathrm{H}_{\mathrm{eq}}-58,{ }^{2} \mathrm{~J}=11.7 \mathrm{~Hz}$,), 3.86 (d, $\left.8 \mathrm{H}, \mathrm{H}_{\mathrm{ax}}-6, \mathrm{H}_{\mathrm{ax}}-16, \mathrm{H}_{\mathrm{ax}}-29, \mathrm{H}_{\mathrm{ax}}-39, \mathrm{H}_{\mathrm{ax}}-47, \mathrm{H}_{\mathrm{ax}}-51, \mathrm{H}_{\mathrm{ax}}-54, \mathrm{H}_{\mathrm{ax}}-58,{ }^{2} \mathrm{~J}=11.7 \mathrm{~Hz}\right), 7.58(\mathrm{~s}, 8 \mathrm{H}$, H-22, H-23, H-45, H-46, H-52, H-53, H-59, H-60), 7.63 (d, 4H, H-10, H-12, H-33, H-35, ${ }^{3} J$ $=7.5 \mathrm{~Hz}), 7.94\left(\mathrm{t}, 2 \mathrm{H}, \mathrm{H}-11, \mathrm{H}-34,{ }^{3} \mathrm{~J}=7.5 \mathrm{~Hz}\right)$.

${ }^{13}$ C NMR (75 MHz, $\left.\mathrm{CDCl}_{3}\right): \delta 19.2\left(\mathrm{CH}_{3}-5, \mathrm{CH}_{3}-17, \mathrm{CH}_{3}-28, \mathrm{CH}_{3}-40\right), 27.8\left(\mathrm{CH}_{3}-8, \mathrm{CH}_{3}-14\right.$, $\left.\mathrm{CH}_{3}-31, \mathrm{CH}_{3}-37\right), 33.8$ (C-5, C-17, C-28, C-40), 67.5 (C-6, C-16, C-29, C-39, C-47, C-51, C54, C-58), 68.1 (C-4, C-18, C-27, C-41), 100.3 (C-8, C-14, C-31, C-37), 120.7 (C-10, C-12, C-33, C35), 129.4 (C-22, C-23, C-45, C-46, C-52, C-53, C-59, C-60), 133.6 (C-1, C-21, C-24, C-44), 137.7 (C-11, C-34), 160.3 (C-9, C-13, C-32, C-36), 164.7 (C-2, C-20, C-25, C-43).

MS (IC positive, $200 \mathrm{eV}$, gaze: isobutane) (rel. int., \%) m/z: $995\left(\mathrm{MH}^{+}, 9\right), 337$ (9), 295 (100), 263 (14), 185 (7), 137 (10), 121 (27), 101 (14).

Calculated for $\mathrm{C}_{54} \mathrm{H}_{62} \mathrm{~N}_{2} \mathrm{O}_{16}$ : C 65.18, H 6.28, N 2.82, found C 65.27, H 6.15, N 2.95 


\section{5,8,14,17-tetramethyl-2,20-dioxo-27,30-diaza-3,7,15,19,26,28-hexaoxa-}

pentacyclo[19.3.2 $\left.2^{5,8} \cdot 2^{14,17} \cdot 1^{9,13} \cdot 1^{1,21}\right]$ triaconta-1(24),9(27),10,12,21(30),22-hexaene (5a).

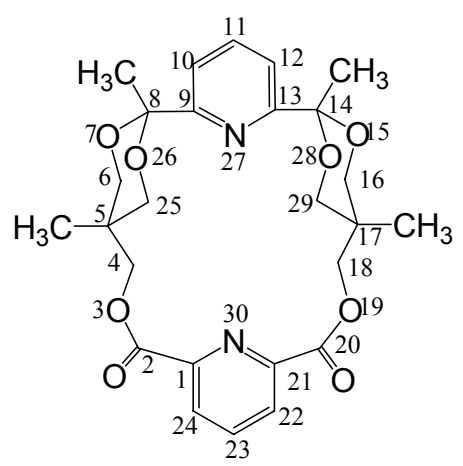

Yield: $52 \%$, white crystals; m.p. T $>310{ }^{\circ} \mathrm{C}$ (decomposition) ${ }^{\mathbf{1}} \mathbf{H} \mathbf{N M R}\left(500 \mathrm{MHz}, \mathrm{CDCl}_{3}, 253\right.$ $\mathrm{K}): \delta 1.41\left(\mathrm{~s}, 6 \mathrm{H}, \mathrm{CH}_{3 \mathrm{ax}}-5, \mathrm{CH}_{3 \mathrm{ax}}-17\right), 1.55$ (s, 6H, $\left.\mathrm{CH}_{3 \mathrm{eq}}-8, \mathrm{CH}_{3 \mathrm{eq}}-14\right), 3.61$ (dd, 2H, $\mathrm{H}_{\mathrm{eq}}-6$, $\left.\mathrm{H}_{\mathrm{eq}}-16,{ }^{2} \mathrm{~J}=11.5 \mathrm{~Hz},{ }^{4} \mathrm{~J}=2.0 \mathrm{~Hz}\right), 3.64$ (d, 2H, CHH-4, CHH-18, $\left.{ }^{2} \mathrm{~J}=12.0 \mathrm{~Hz}\right), 3.73$ (d, 2H, $\left.\mathrm{H}_{\mathrm{ax}}-6, \mathrm{H}_{\mathrm{ax}}-16,{ }^{2} \mathrm{~J}=11.5 \mathrm{~Hz}\right), 3.74\left(\mathrm{dd}, 2 \mathrm{H}, \mathrm{H}_{\mathrm{eq}}-25, \mathrm{H}_{\mathrm{eq}}-29,{ }^{2} \mathrm{~J}=11.5 \mathrm{~Hz},{ }^{4} \mathrm{~J}=2.0 \mathrm{~Hz}\right), 4.07$ (d, 2H, $\left.\mathrm{H}_{\mathrm{ax}}-25, \mathrm{H}_{\mathrm{ax}}-29,{ }^{2} \mathrm{~J}=11.5 \mathrm{~Hz}\right), 4.89$ (d, 2H, CHH-4, CHH-18 $\left.{ }^{2} \mathrm{~J}=12.0 \mathrm{~Hz}\right), 7.31$ (d, $\left.2 \mathrm{H}, \mathrm{H}-10, \mathrm{H}-12,{ }^{3} \mathrm{~J}=7.5 \mathrm{~Hz}\right), 7.53$ (t, $\left.1 \mathrm{H}, \mathrm{H}-11,{ }^{3} \mathrm{~J}=7.5 \mathrm{~Hz}\right), 8.05$ (t, $1 \mathrm{H}, \mathrm{H}-23,{ }^{3} J=8.0 \mathrm{~Hz}$ ) $8.31\left(\mathrm{~d}, 2 \mathrm{H}, \mathrm{H}-22, \mathrm{H}-24,{ }^{3} \mathrm{~J}=8.0 \mathrm{~Hz}\right)$.

${ }^{13}$ C NMR $\left(75 \mathrm{MHz}, \mathrm{CDCl}_{3}\right): \delta 19.1\left(\mathrm{CH}_{3}-5, \mathrm{CH}_{3}-17\right), 30.7\left(\mathrm{CH}_{3}-8, \mathrm{CH}_{3}-14\right), 35.0(\mathrm{C}-5, \mathrm{C}-$ 17), 67.5 (C-4, C-18), 67.7 (C-6, C-16, C-25, C-29), 100.5 (C-8, C-14), 120.7 (C-10, C-12), 128.6 (C-22, C-24), 137.4(C-11), 138.3 (C-23), 147.5 (C-1, C21) 160.0 (C-9, C-13), 164.5 (C-2, C-20).

MS (EI, 70 eV) (rel. int., \%) m/z: 498 (M+, 6), 469 (2), 427 (8), 355 (3), 249 (11), 204 (8), 150 (14), 122 (41), 85 (31), 55 (100).

Calculated for $\mathrm{C}_{26} \mathrm{H}_{30} \mathrm{~N}_{2} \mathrm{O}_{8}$ : C 62.64, H 6.07, N 5.62; found C 62.79, H 6.15, N 5.78

Complex 6a: $\left[\left(\mathrm{C}_{26} \mathrm{H}_{30} \mathrm{~N}_{2} \mathrm{O}_{8}\right)_{2} \mathrm{Ag}_{4}\right](\mathrm{OTf})_{4} \cdot \mathrm{H}_{2} \mathrm{O}$

Yield: $85 \%$, white crystals;

${ }^{1} \mathbf{H}$ NMR $\left(\mathrm{CDCl}_{3}, 300 \mathrm{MHz}\right) \delta \mathrm{ppm} ; 0.91$ (s, 6H), 1.77 (s, 6H), 4.15-4.45 (broad signals), 7.31-7.30 (m, 3H), 8.03 (t, 1H, $J=10.9 \mathrm{~Hz}), 8.36$ (d, 2H, $J=10.9 \mathrm{~Hz})$.

${ }^{13}$ C NMR $\left(62.5 \mathrm{MHz}, \mathrm{CDCl}_{3}\right) \delta \mathrm{ppm}:$ 18.6, 30.6, 34.7, 66.3, 67.6, 69.3, 77.4, 99.6, 122.8, $128.6,129.3,129.8,135.0,140.4,159.6,164.7$

Calculated for: $\left[\left(\mathrm{C}_{26} \mathrm{H}_{30} \mathrm{~N}_{2} \mathrm{O}_{8}\right)_{2} \mathrm{Ag}_{4}\right](\mathrm{OTf})_{4} \cdot 4 \mathrm{H}_{2} \mathrm{O}: \mathrm{C} 32.06, \% \mathrm{H} \mathrm{3.24,} \mathrm{\%} \mathrm{N} \mathrm{2.67,} \mathrm{\%} 6.11, \% \mathrm{~F}$ 10.88, \% Ag 20.61; found: C 32.17, \% H 3.11, \% N 2.45, \%S 6.33, \%F 10.99, \%Ag 20.79 


\section{2,6-bis (2,5-dimethyl-5-hydroxymethyl-1,3-dioxane-2-yl)pyridine (7)}

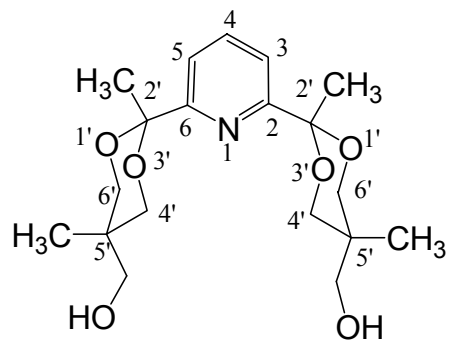

Yield: $16 \%$, white crystals; m.p.: $186-187{ }^{\circ} \mathrm{C}$; purified by flash chromatography (silica, $\mathrm{DCM} /$ methanol/ammonia $=4.7 / 0.3 / 0.01) . \mathrm{R}_{f}=0.3$.

${ }^{1}$ H NMR (300 MHz, $\left.\mathrm{CDCl}_{3}\right): \delta 1.13$ (s, 6H, $\mathrm{CH}_{3 \mathrm{ax}}-5$ ') 1.67 (s, 6H, $\mathrm{CH}_{3 \mathrm{eq}}-2$ ') $), 3.35$ (s, 4H, $\left.\underline{\mathrm{CH}}_{2}-\mathrm{OH}\right), 3.61$ (d, 4H, Heq $\left.-4^{\prime}, \mathrm{H}_{\mathrm{eq}}-6^{\prime},{ }^{2} J=11.5 \mathrm{~Hz}\right), 3.76\left(\mathrm{~d}, 4 \mathrm{H}, \mathrm{H}_{\mathrm{ax}}-4^{\prime}, \mathrm{H}_{\mathrm{ax}}-6^{\prime},{ }^{2} J=11.5\right.$ $\mathrm{Hz}$ ), 7.50 (d, 2H, H-3, H-5, $\left.{ }^{3} \mathrm{~J}=7.9 \mathrm{~Hz}\right), 7.76$ (t, $1 \mathrm{H}, \mathrm{H}-4{ }^{3} \mathrm{~J}=7.9 \mathrm{~Hz}$ ).

${ }^{13}$ C NMR (75 MHz, CD $\left.{ }_{3} \mathrm{OD}\right): \delta: \delta 18.8\left(\mathrm{CH}_{3}-5^{\prime}\right), 28.1\left(\mathrm{CH}_{3}-2^{\prime}\right), 36.1\left(\mathrm{C}-5\right.$ ') $66.7\left(\mathrm{CH}_{2} \mathrm{OH}\right)$, 69.2 (C-4', C-6'), 101.2 (C-2'), 122.0 (C-3, C-5), 139.2 (C-4), 161.2 (C-2, C-6).

MS (EI, $70 \mathrm{eV})(\mathrm{rel}$ int, \%) m/z: 367 (M+1, 8), 266 (80), 224 (5), 164(91), 145(100), 85(49), $57(35)$.

Calculated for $\mathrm{C}_{19} \mathrm{H}_{29} \mathrm{NO}_{6}$ : C 61.11, H 7.96, N 3.81, found C 60.95, H 7.86, N 3.89. 


\subsection{Copy of ${ }^{1} \mathrm{H}$ and ${ }^{13} \mathrm{C}$ NMR spectra of compounds $2-7$.}

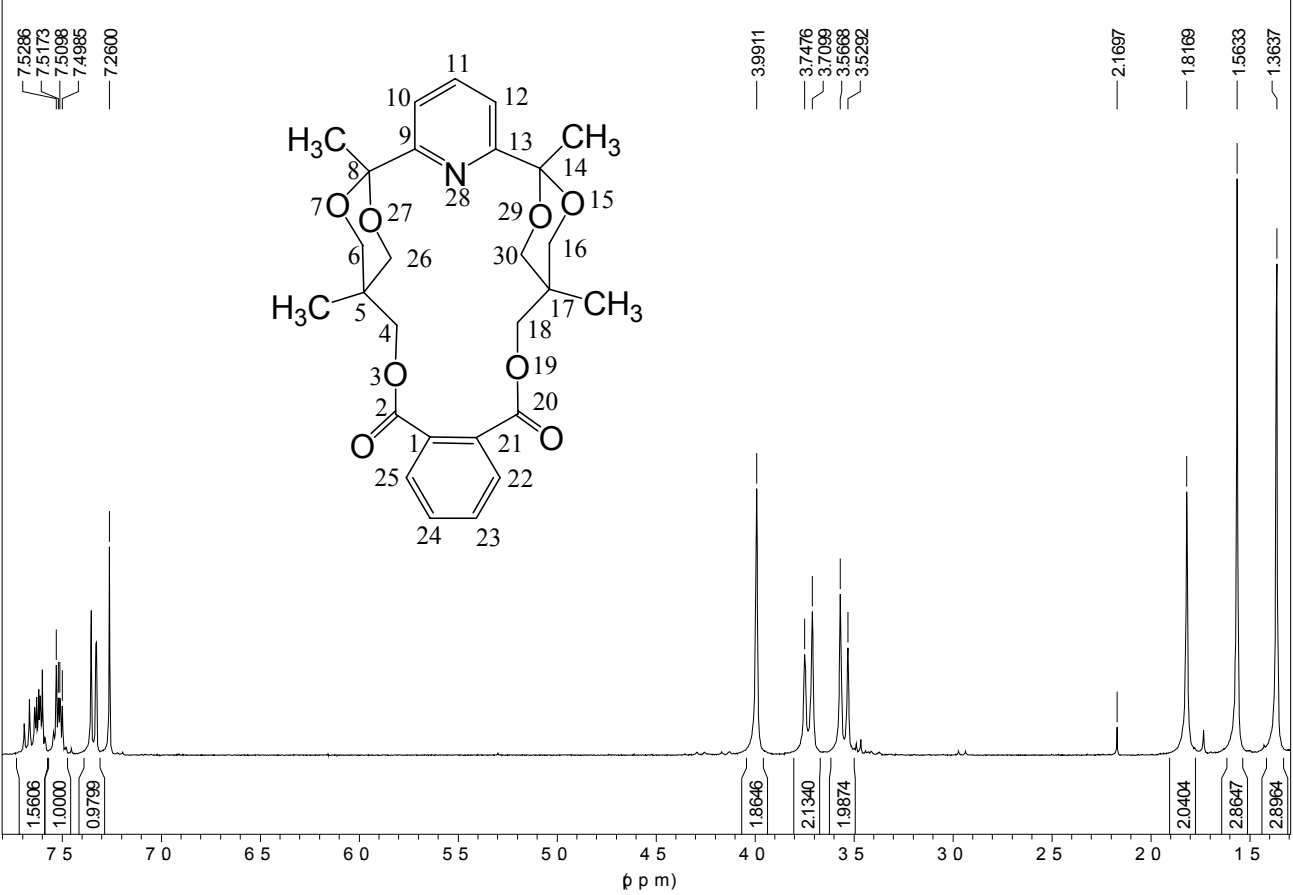

Figure $9{ }^{1} \mathrm{H}-\mathrm{NMR}$ spectra of compound 2a at $r t$

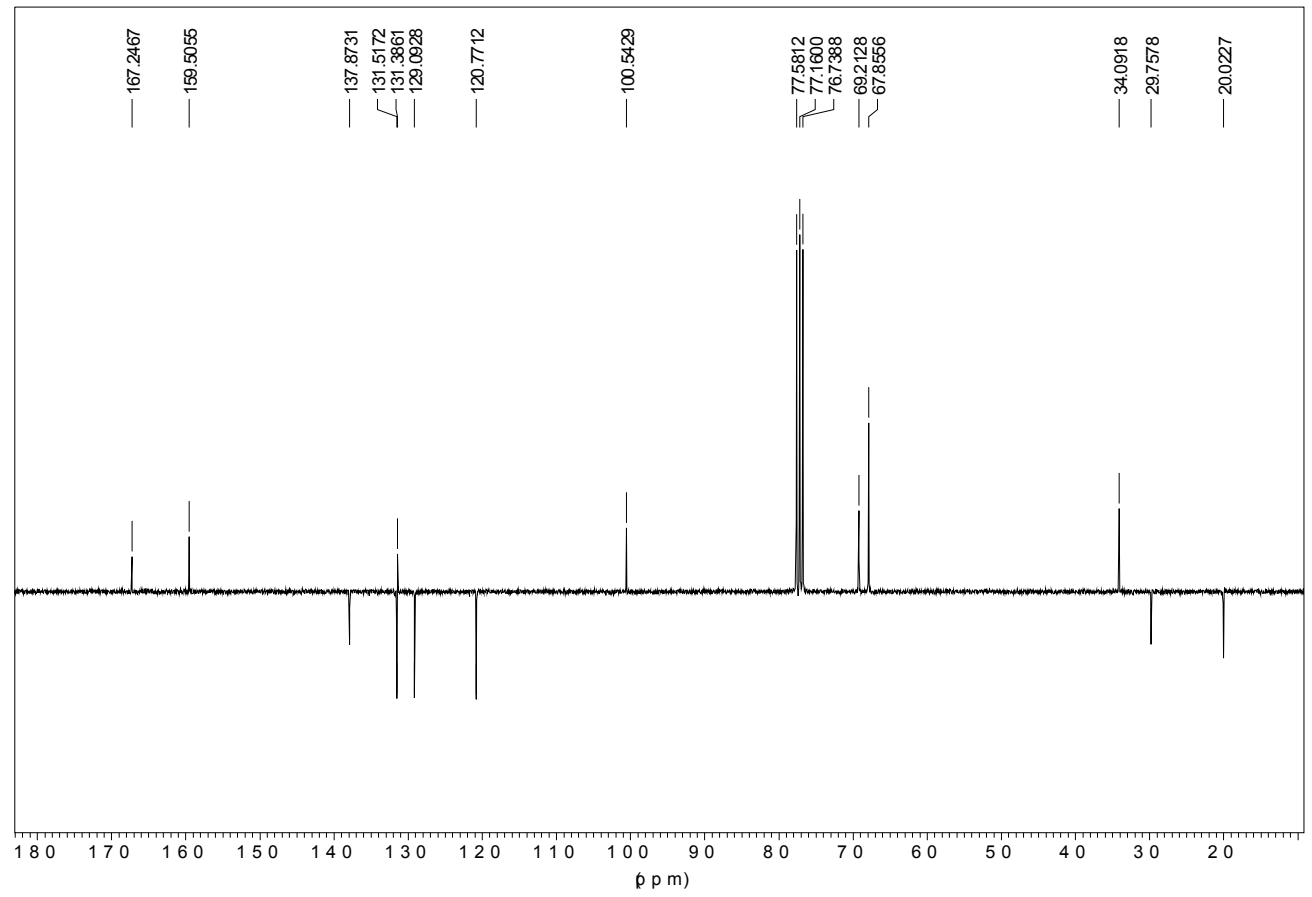

Figure $10{ }^{13} \mathrm{C}$-NMR spectra of compound $\mathbf{2 a}$ at $r t$ 


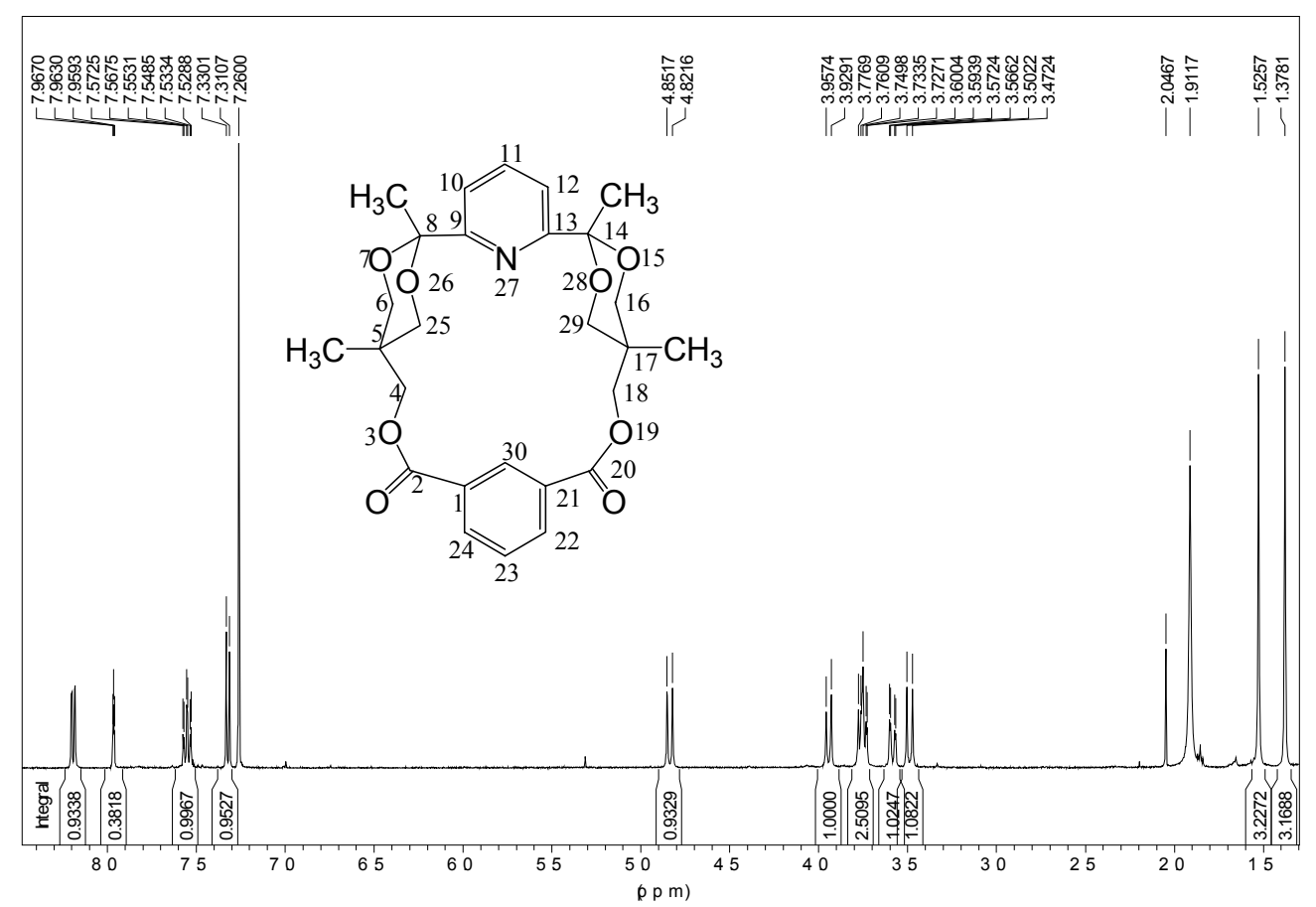

Figure 11. ${ }^{1} \mathrm{H}-\mathrm{NMR}$ spectra of compound 3a at $253 \mathrm{~K}$

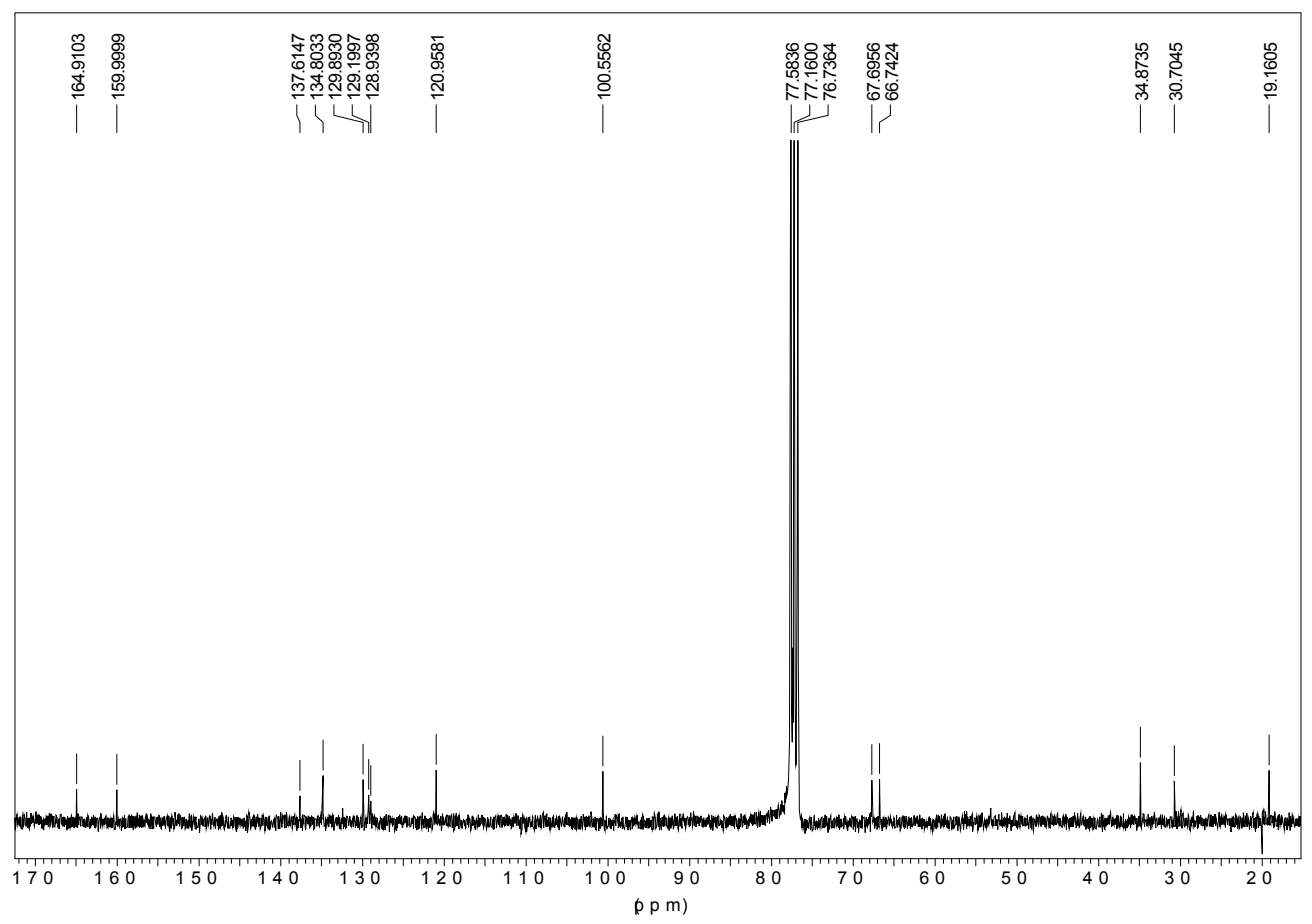

Figure 12. ${ }^{13} \mathrm{C}-\mathrm{NMR}$ spectra of compound 3a at $r t$ 


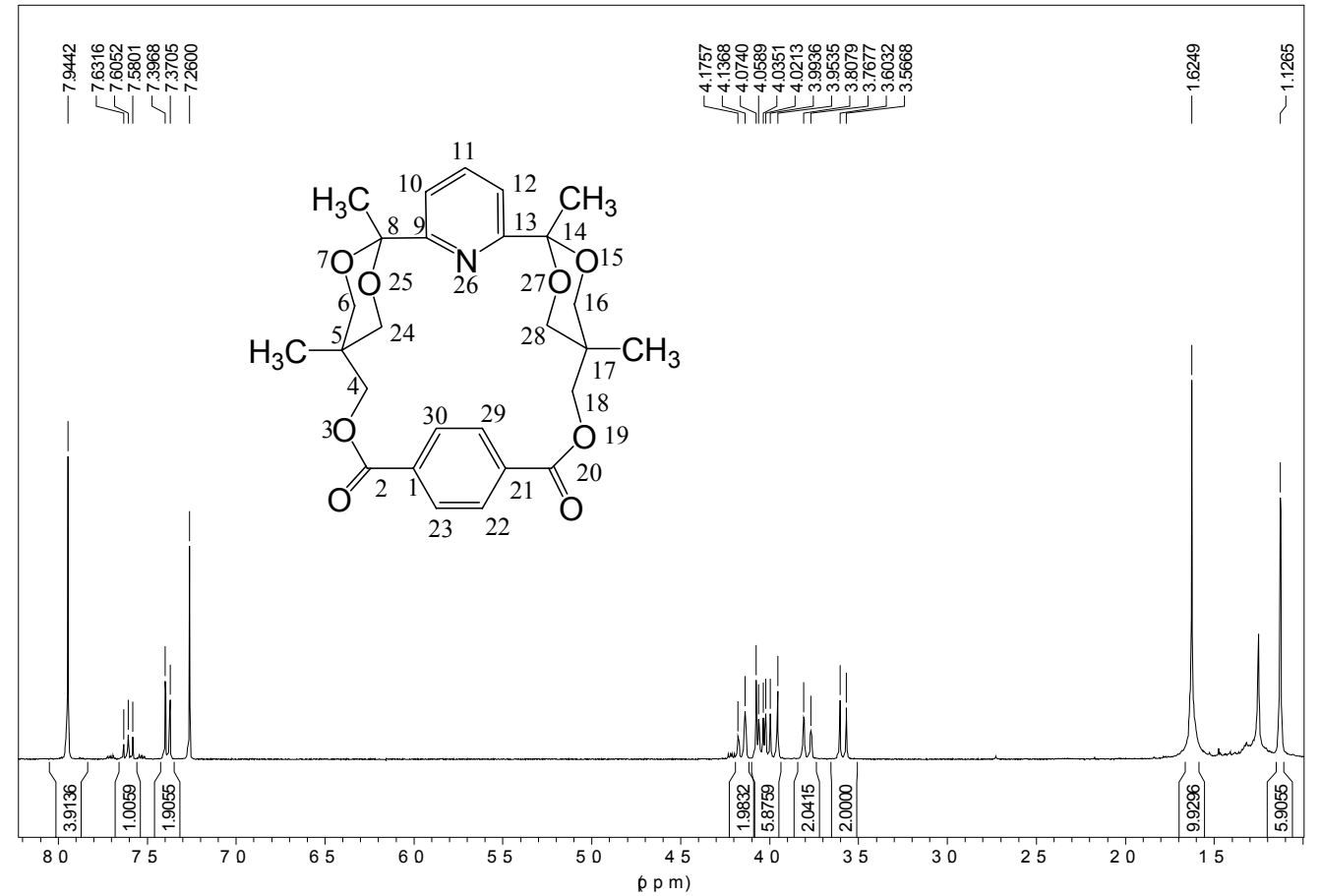

Figure 13. ${ }^{1} \mathrm{H}-\mathrm{NMR}$ spectra of compound $\mathbf{4 a}$ at $r t$

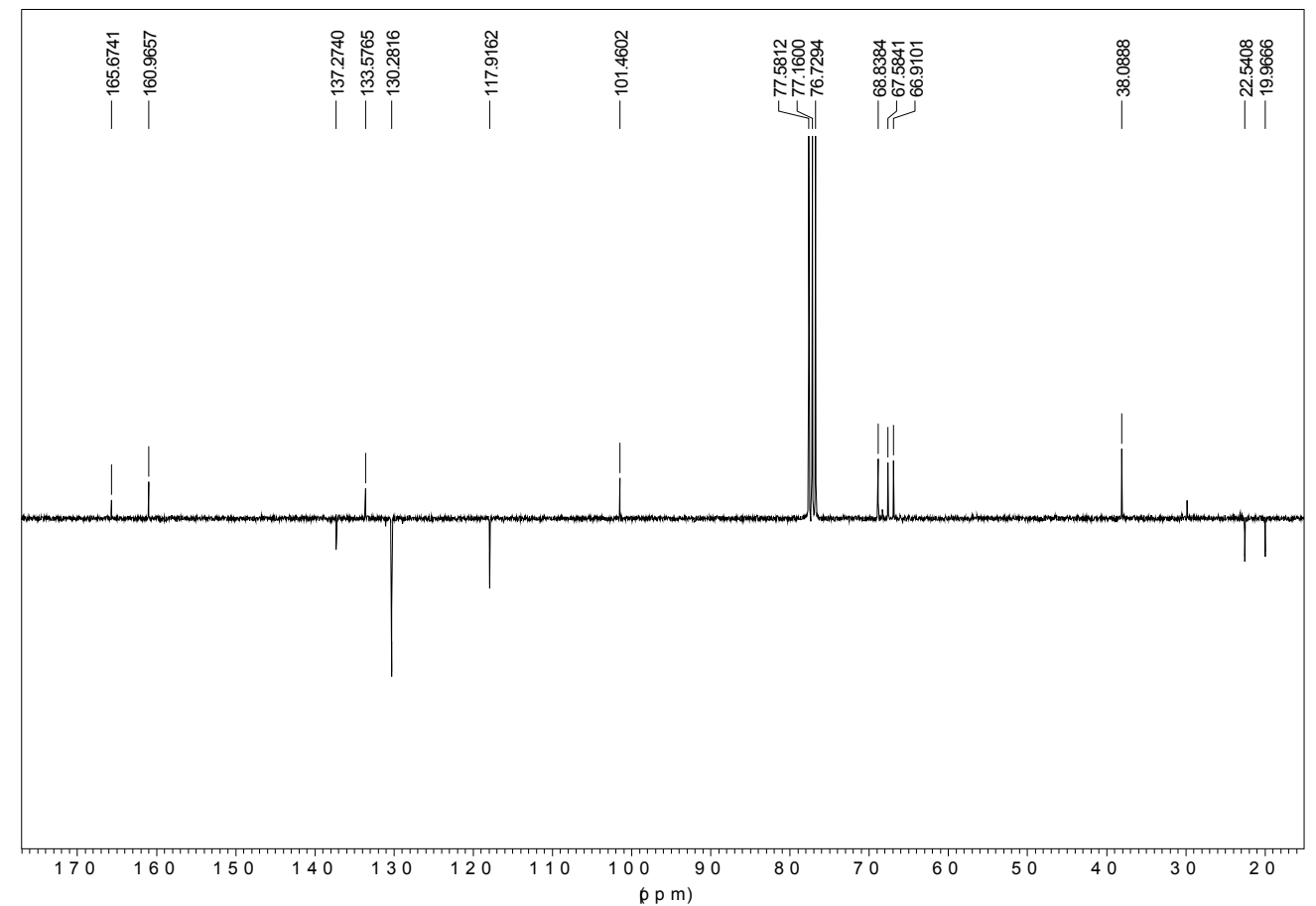

Figure 14. ${ }^{13} \mathrm{C}$-NMR spectra of compound $4 \mathbf{a}$ at $r t$ 


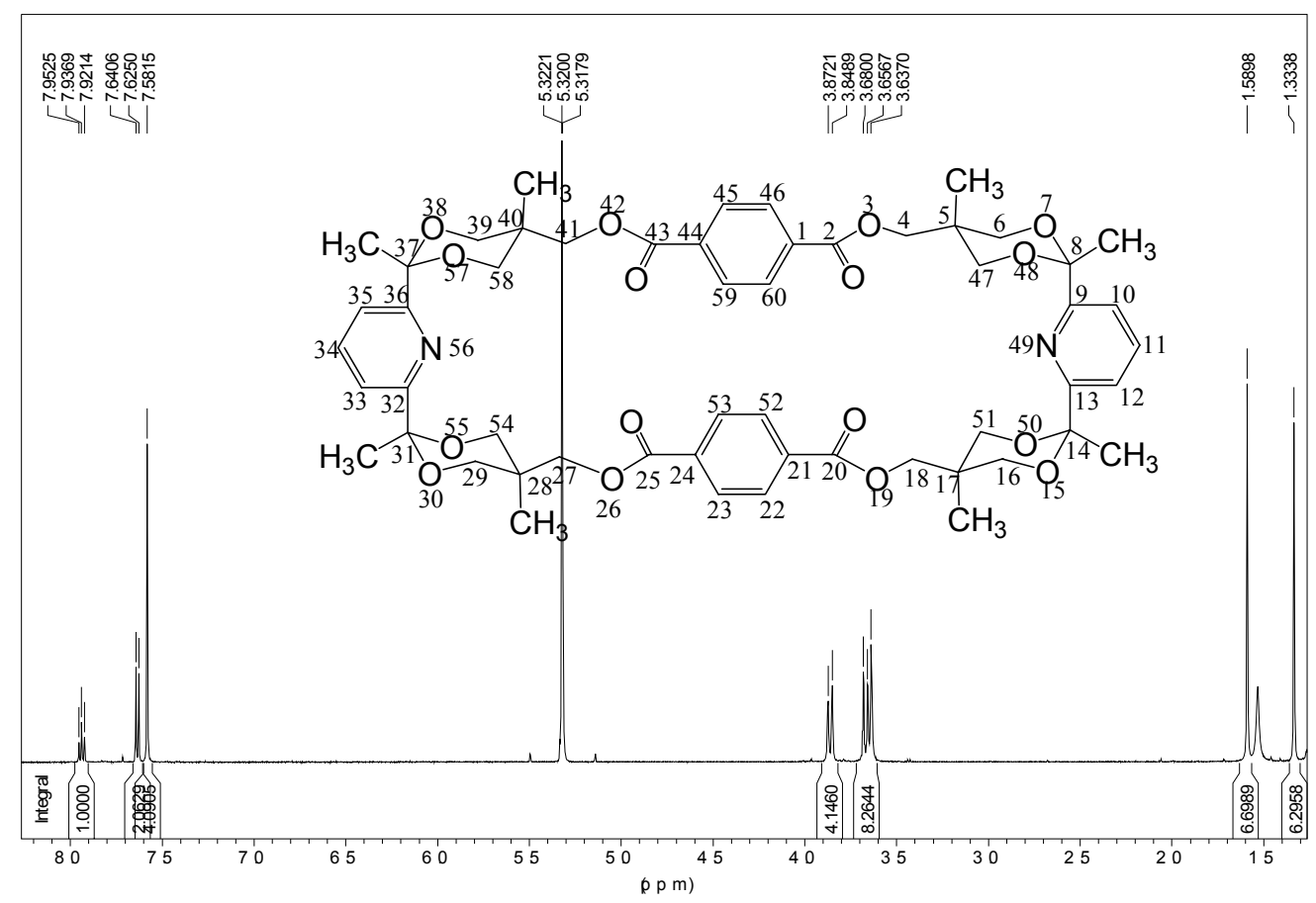

Figure 15. ${ }^{1} \mathrm{H}-\mathrm{NMR}$ spectra of compound $\mathbf{4 b}$ at $r t$.

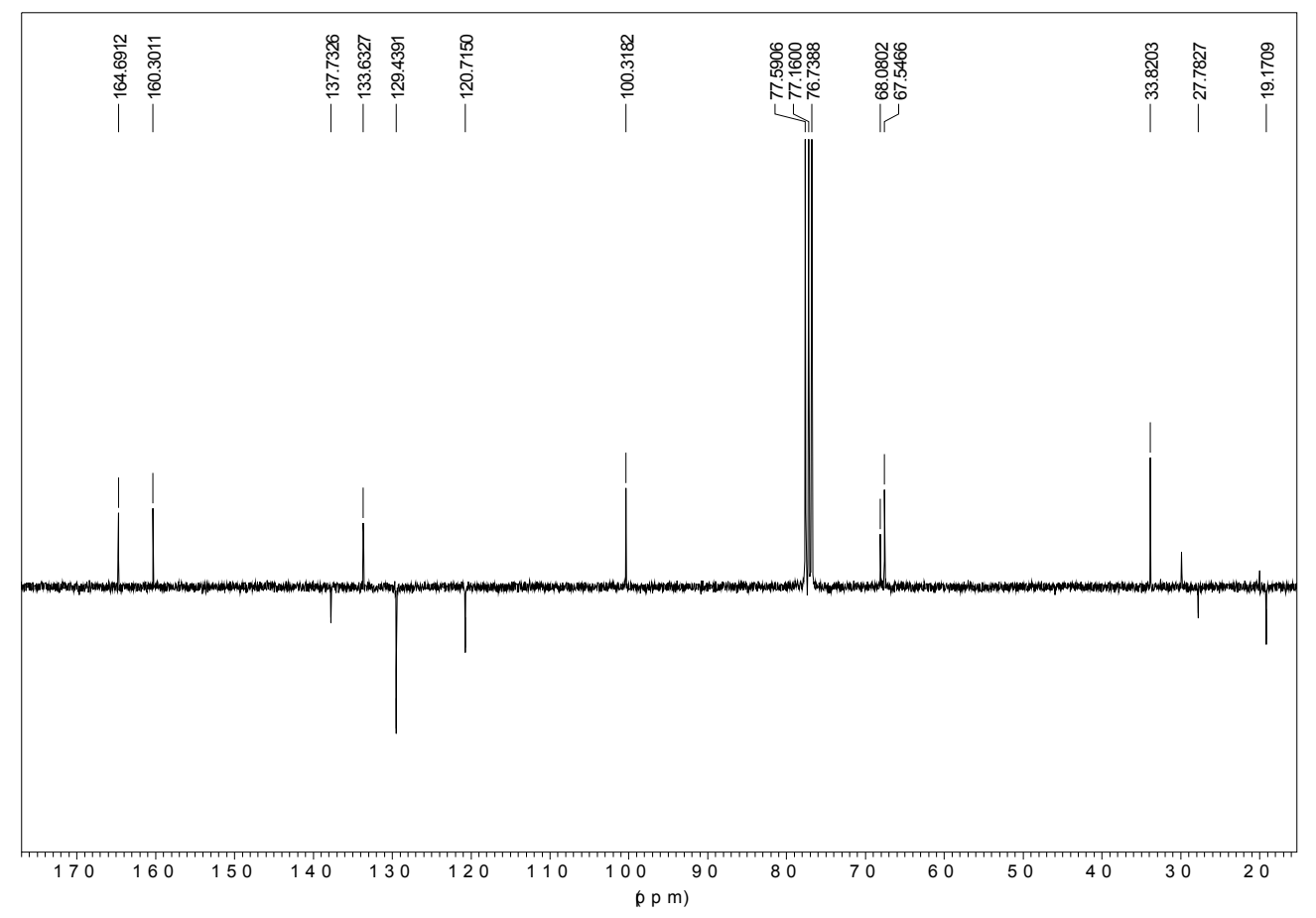

Figure 16. ${ }^{13} \mathrm{C}$-NMR spectra of compound $\mathbf{4 b}$ at $r t$ 


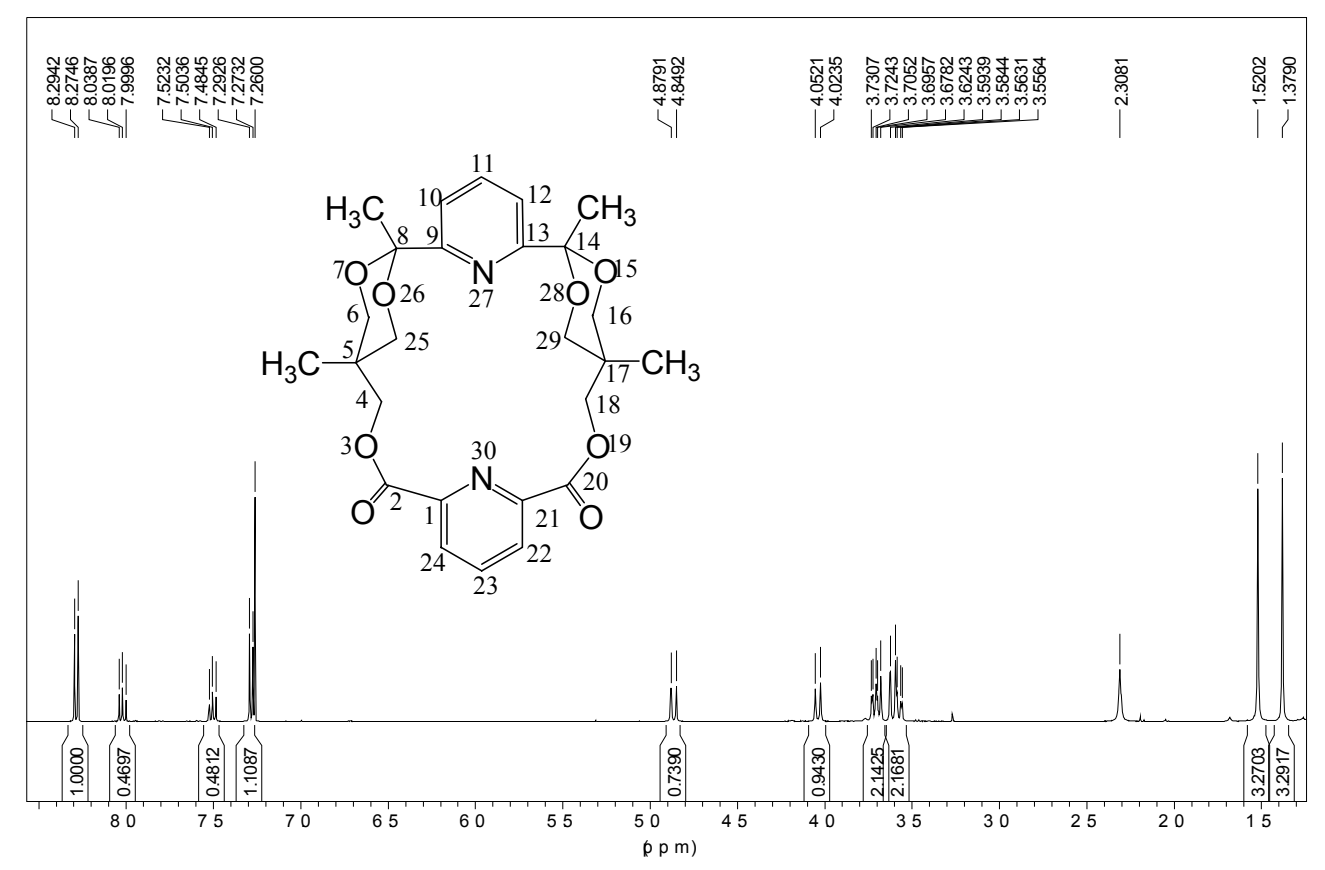

Figure 17. ${ }^{1} \mathrm{H}-\mathrm{NMR}$ spectra of compound 5a at $253 \mathrm{~K}$

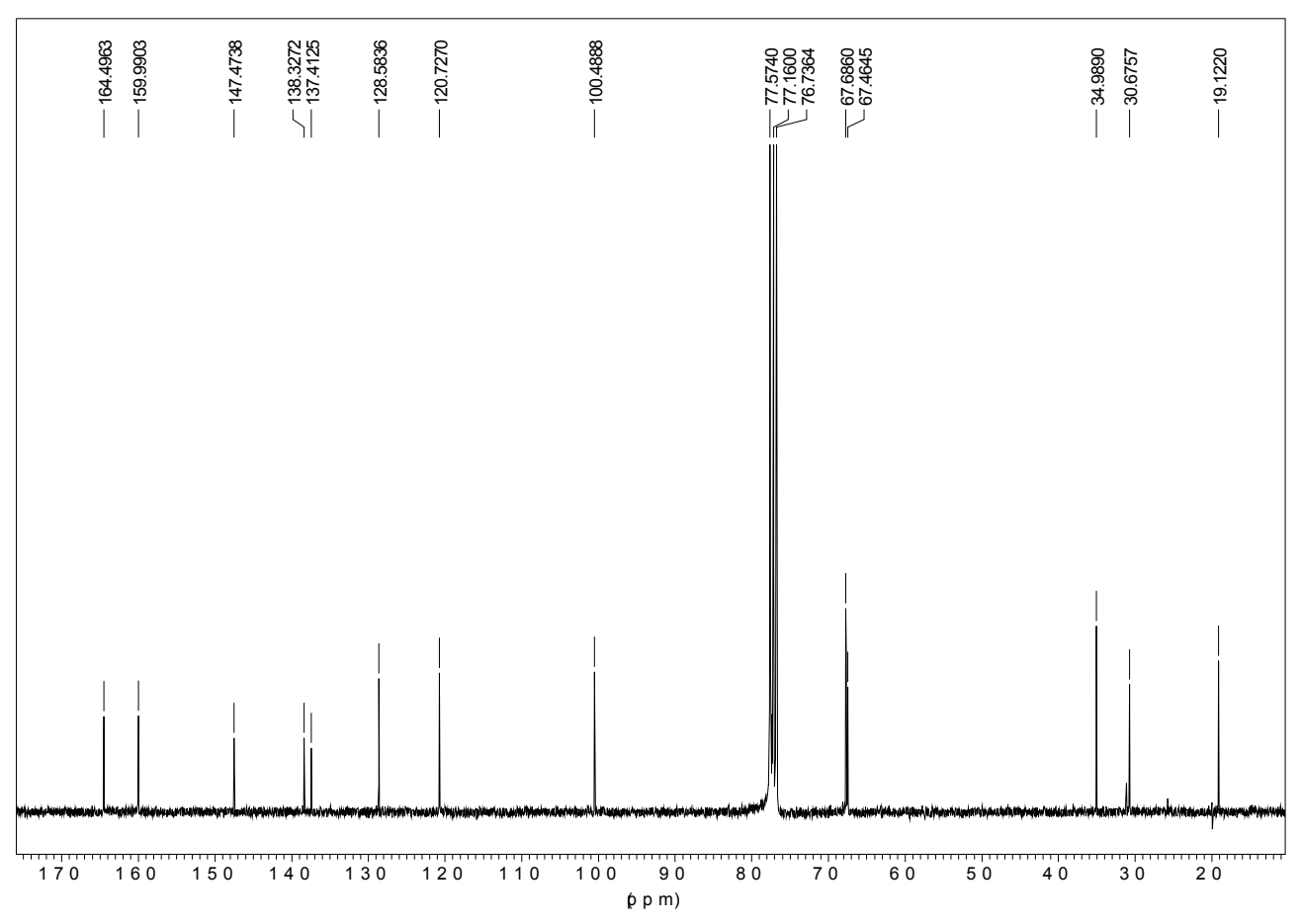

Figure 18. ${ }^{13} \mathrm{C}$-NMR spectra of compound 5a at $r t$ 


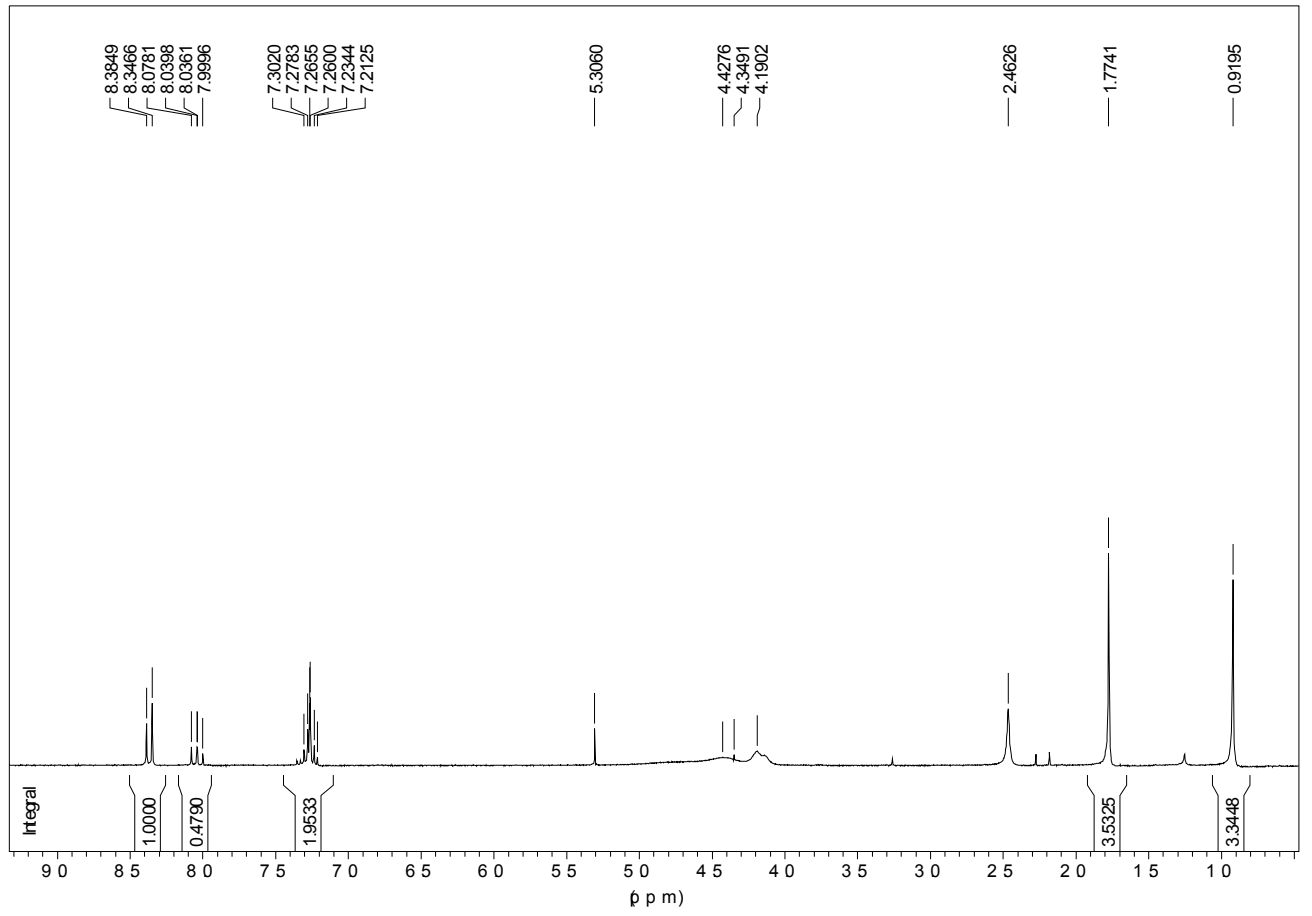

Figure 19. ${ }^{1} \mathrm{H}-\mathrm{NMR}$ spectra of compound $\mathbf{6 a}$ at $r t$

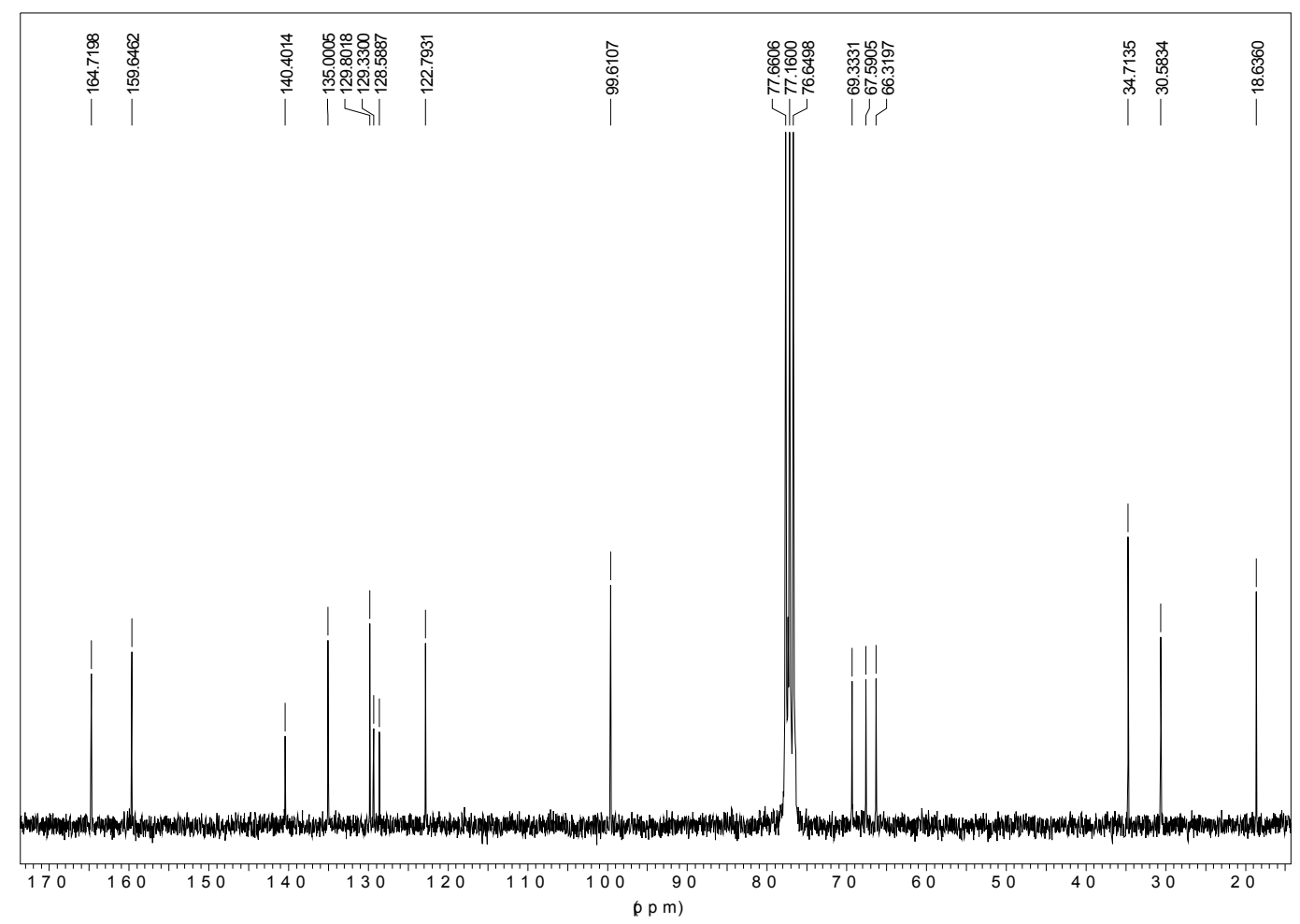

Figure 20. ${ }^{13} \mathrm{C}$-NMR spectra $(62.5 \mathrm{MHz})$ of compound $6 \mathbf{a}$ 


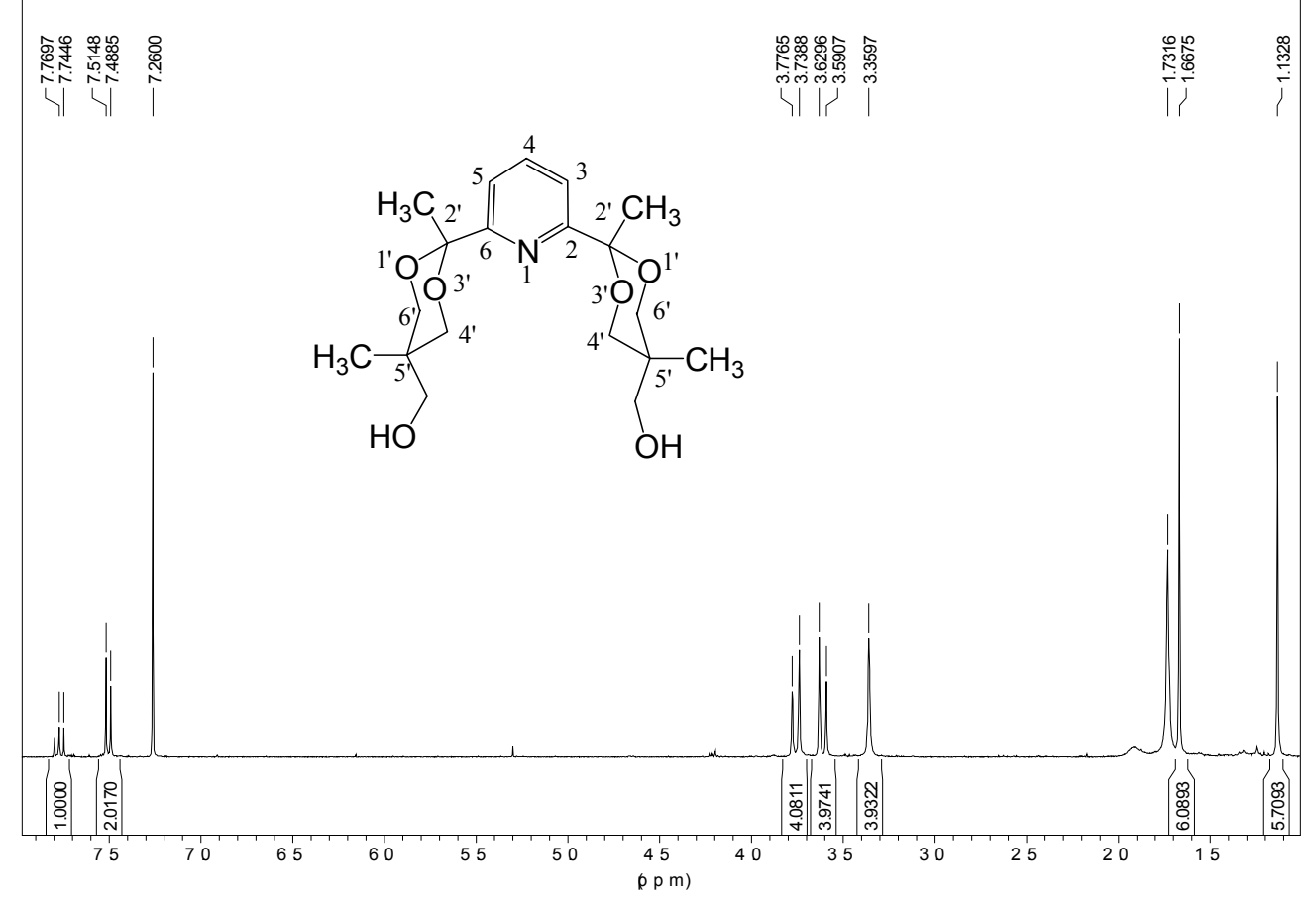

Figure 21. ${ }^{1} \mathrm{H}-\mathrm{NMR}$ spectra of compound 7

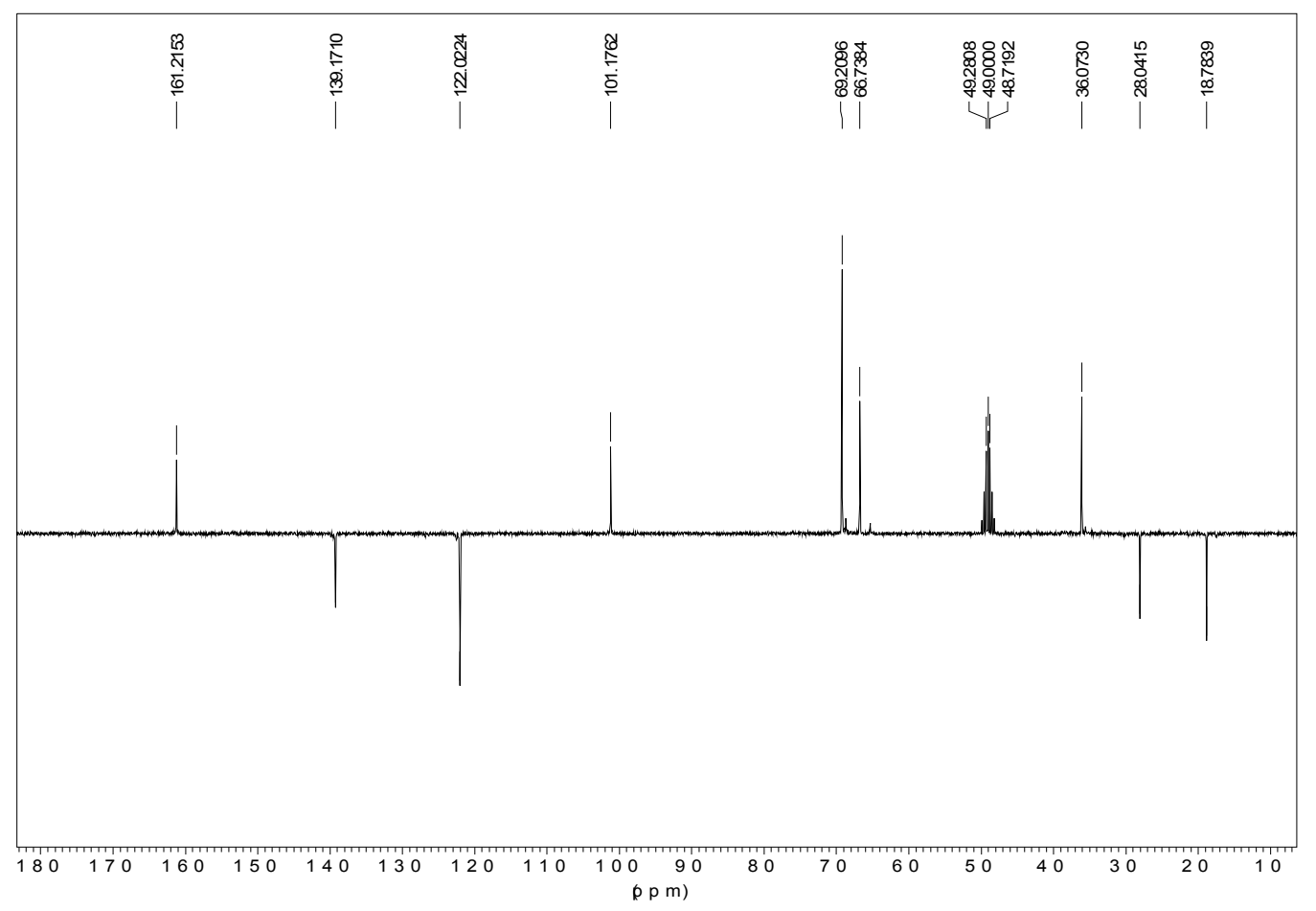

Figure 22. ${ }^{13} \mathrm{C}-\mathrm{NMR}$ spectra of compound 7 


\subsection{Parameters of the crystalographic determinations for compound $2 \mathrm{a}, 5 \mathrm{a}, \mathbf{6 a}$.}

Table 6. Parameters of the crystalographic determinations ${ }^{\text {a }}$ for compound $\mathbf{2 a}, \mathbf{5 a}, \mathbf{6 a}$.

\begin{tabular}{|l|l|l|l|}
\hline Compound & $\mathbf{2 a}$ & $\mathbf{5 a}$ & $\mathbf{6 a}$ \\
\hline Empirical formula & $\mathrm{C}_{116} \mathrm{H}_{144} \mathrm{~N}_{4} \mathrm{O}_{34}$ & $\mathrm{C}_{13} \mathrm{H}_{15} \mathrm{NO}_{4}$ & $\mathrm{C}_{29} \mathrm{H}_{35} \mathrm{Ag}_{2} \mathrm{Cl}_{3} \mathrm{~F}_{6} \mathrm{~N}_{2} \mathrm{O}_{16} \mathrm{~S}_{2}$ \\
\hline Formula weight & 2138.35 & 249.26 & 1167.80 \\
\hline Temperature (K) & $120(1)$ & $293(2)$ & $293(2)$ \\
\hline Wavelength, $\AA$ & 0.71073 & 0.71069 & 0.71073 \\
\hline Crystal system & Monoclinic & Monoclinic & Triclinic \\
\hline Space group & $\mathrm{P} 21 / \mathrm{n}$ & $\mathrm{P} 21 / \mathrm{m}$ & $\mathrm{P}-1$ \\
\hline $\mathrm{a}, \AA$ & $18.5086(4)$ & $6.34170(10)$ & $12.2013(2)$ \\
\hline $\mathrm{b}, \AA$ & $28.6914(6)$ & $20.5313(3)$ & $12.9722(2)$ \\
\hline $\mathrm{c}, \AA$ & $22.5474(5)$ & $9.4744(2)$ & $14.3899(3)$ \\
\hline$\alpha^{\circ}$ & 90 & 90 & $90.8577(10)$ \\
\hline$\beta^{\circ}$ & $110.4520(10)$ & 96.3290 & $92.2360(10)$ \\
\hline$\gamma^{\circ}$ & 90 & 90 & $116.5660(10)$ \\
\hline Volume, $\AA^{3}$ & $11218.8(4)$ & $1226.08(4)$ & $2034.24(6)$ \\
\hline$Z$ & 4 & 4 & 2 \\
\hline Density (calculated) $\mathrm{mg} / \mathrm{m}^{3}$ & 1.266 & 1.350 & 1.907 \\
\hline Absorption coefficient, $\mathrm{mm}^{-1}$ & 0.093 & 0.101 & 1.361 \\
\hline
\end{tabular}




\begin{tabular}{|l|l|l|l|}
\hline $\mathrm{F}(000)$ & 4560 & 528 & 1164 \\
\hline Crystal size/mm & $0.25 \times 0.20 \times 0.10$ & $0.22 \times 0.18 \times 0.12$ & $0.30 \times 0.30 \times 0.25$ \\
\hline Theta range for data collection/ $\left(^{\circ}\right)$ & 2.34 to 26.00 & 1.98 to 26.98 & 1.42 to 27.00 \\
\hline Index ranges & $0<\mathrm{H}>22 ; 0<\mathrm{K}>35 ;-27<\mathrm{L}>26$ & $-8<\mathrm{H}>7 ;-26<\mathrm{K}>26 ;-12<\mathrm{L}>12$ & $-15<\mathrm{H}>14 ;-16<\mathrm{K}>16 ;-18<\mathrm{L}>18$ \\
\hline Reflections collected & 21936 & 20517 & 17700 \\
\hline Independent reflections & $21936\left(\mathrm{R}_{\text {int }}=0.0000\right)$ & $2743\left(\mathrm{R}_{\text {int }}=0.0364\right)$ & $8098\left(\mathrm{R}_{\text {int }}=0.0196\right)$ \\
\hline Refinement method & Full matrix least-square on $\mathrm{F}^{2}$ & Full matrix least-square on $\mathrm{F}^{2}$ & Full matrix least-square on $\mathrm{F}^{2}$ \\
\hline Data/restraints/parameters & $21936 / 0 / 13872773$ & $2743 / 0 / 170$ & $8098 / 0 / 553$ \\
\hline Goodness-of-method on $\mathrm{F}^{2}$ & 1.088 & 1.100 & 1.066 \\
\hline Final $\mathrm{R}$ indices [I $>2 \sigma(\mathrm{I})]$ & $\mathrm{R} \sim 1=0.079, \mathrm{wR} \sim 2=0.1749$ & $\mathrm{R} \sim 1=0.0493, \mathrm{wR} \sim 2=0.1584$ & $\mathrm{R} \sim 1=0.0258, \mathrm{wR} \sim 2=0.0698$ \\
\hline $\mathrm{R}$ indices $($ all data) & $\mathrm{R} \sim 1=0.1528, \mathrm{wR} \sim 2=0.2292$ & $\mathrm{R} \sim 1=0.0683, \mathrm{wR} \sim 2=0.1903$ & $\mathrm{R} \sim 1=0.0282, \mathrm{wR} \sim 2=0.0726$ \\
\hline Largest diff. peack on hole, $\mathrm{eA}^{3}$ & $<0.9$ & $<1.388$ & $<1.891$ \\
\hline
\end{tabular}

The sample is studied on a NONIUS Kappa CCD diffractometer with graphite monochromatized MoKa radiation for 2a or on a Bruker AXS X8-APEX II with graphite monochromatized MoKa radiation for $\mathbf{5 a}$ and $\mathbf{6 a}$.The structure was solved with SIR-97 ${ }^{10}$ which reveals the non hydrogen atoms of the molecule. After anisotropic refinement, many hydrogen atoms may be found with a Fourier Difference. The whole structure was refined with SHELXL9 $7^{11}$ by the full-matrix least-square techniques. Atomic scattering factors from International Tables for Xray Crystallography (1992). Ortep views realized with PLATON98.

(10)Altomare, M. C. Burla, M. Camalli, G. Cascarano, C. Giacovazzo, A. Guagliardi, A.G.G. Moliterni, G. Polidori, R. Spagna. Sir97: a new tool for crystal structure determination and refinement. Jr of Applied Crystallography, 1998, 31, 74-77.

(11) Sheldrick, G.M. (1997).SHELX97. Program for the Refinement of Crystal Structures, Univ. of Göttingen, Germany. 
5.8 Cartesian coordinates of the B3lyp/6-31G(d) optimized structures of 2a-5a.

Compound: 2a-syn

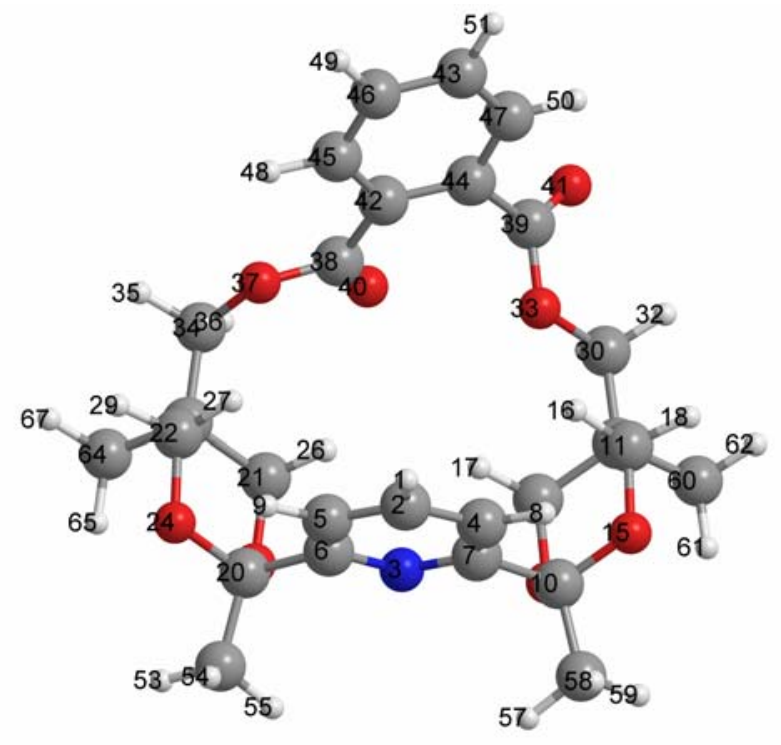

$\begin{array}{lrrr}\text { Atom } & \mathbf{X} & \mathbf{Y} & \mathbf{Z} \\ 1 & 1.435398 & 0.482588 & 4.096798 \\ 6 & 1.665508 & 0.658777 & 3.049049 \\ 7 & 2.260372 & 1.112743 & 0.370395 \\ 6 & 1.244056 & 1.829730 & 2.422691 \\ 6 & 2.383352 & -0.285977 & 2.316898 \\ 6 & 2.650352 & -0.018299 & 0.970327 \\ 6 & 1.568096 & 2.015323 & 1.073450 \\ 1 & 0.680451 & 2.588443 & 2.954839 \\ 1 & 2.733059 & -1.208443 & 2.767386 \\ 6 & 1.223131 & 3.335769 & 0.348537 \\ 6 & -1.165903 & 3.203541 & 0.526872 \\ 6 & 0.017958 & 2.395659 & -1.479930 \\ 6 & -1.297613 & 3.038098 & -1.001452 \\ 8 & 1.136994 & 3.168084 & -1.048390 \\ 8 & 0.028783 & 3.910125 & 0.876850 \\ 1 & -1.180761 & 2.219537 & 1.011800 \\ 1 & 0.098025 & 1.363219 & -1.118768 \\ 1 & -1.988139 & 3.801361 & 0.934798 \\ 1 & 0.075633 & 2.380532 & -2.573056 \\ 6 & 3.503017 & -0.978813 & 0.111941 \\ 6 & 1.855027 & -1.442372 & -1.541959 \\ 6 & 2.169996 & -2.961563 & 0.373555 \\ 6 & 1.773405 & -2.920373 & -1.116169 \\ 8 & 3.425195 & -2.316485 & 0.605123 \\ 8 & 3.148790 & -0.917422 & -1.252378\end{array}$




$\begin{array}{lrrr}1 & 1.079170 & -0.847573 & -1.044557 \\ 1 & 1.383919 & -2.492273 & 0.978620 \\ 1 & 1.722951 & -1.331421 & -2.622353 \\ 1 & 2.302498 & -3.991234 & 0.723475 \\ 6 & -2.495685 & 2.156559 & -1.362570 \\ 1 & -2.484216 & 1.895360 & -2.426573 \\ 1 & -3.439710 & 2.674417 & -1.161046 \\ 8 & -2.473757 & 0.936583 & -0.583637 \\ 6 & 0.359958 & -3.484045 & -1.327600 \\ 1 & 0.375220 & -4.572733 & -1.214171 \\ 1 & -0.011020 & -3.231133 & -2.324625 \\ 8 & -0.600070 & -3.036163 & -0.342491 \\ 6 & -1.550074 & -2.142007 & -0.709044 \\ 6 & -3.556545 & 0.146388 & -0.728906 \\ 8 & -1.555198 & -1.556354 & -1.772966 \\ 8 & -4.486049 & 0.396737 & -1.464443 \\ 6 & -2.606466 & -2.011903 & 0.339634 \\ 6 & -4.738515 & -1.944234 & 2.162625 \\ 6 & -3.569732 & -0.987212 & 0.260426 \\ 6 & -2.720441 & -2.981507 & 1.347280 \\ 6 & -3.771349 & -2.944445 & 2.260228 \\ 6 & -4.638456 & -0.978203 & 1.162797 \\ 1 & -1.983645 & -3.774161 & 1.395375 \\ 1 & -3.842699 & -3.704098 & 3.033299 \\ 1 & -5.391117 & -0.201133 & 1.073966 \\ 1 & -5.571964 & -1.916343 & 2.858887 \\ 6 & 4.973176 & -0.579781 & 0.187478 \\ 1 & 5.559395 & -1.258522 & -0.438066 \\ 1 & 5.329838 & -0.641030 & 1.219273 \\ 1 & 5.090018 & 0.443556 & -0.177620 \\ 6 & 2.330700 & 4.359058 & 0.583192 \\ 1 & 3.274974 & 3.967692 & 0.196183 \\ 1 & 2.434928 & 4.568655 & 1.651202 \\ 1 & 2.078446 & 5.283634 & 0.056816 \\ 6 & -1.497937 & 4.405057 & -1.679856 \\ 1 & -0.640189 & 5.051455 & -1.483678 \\ 1 & -2.398982 & 4.904211 & -1.303618 \\ 1 & -1.600146 & 4.294269 & -2.766401 \\ 6 & 2.740921 & -3.764983 & -1.964916 \\ 1 & 3.753949 & -3.365119 & -1.890578 \\ 1 & 2.448295 & -3.754911 & -3.022288 \\ 1 & 2.756673 & -4.808286 & -1.626916\end{array}$


Compound: 2a-anti

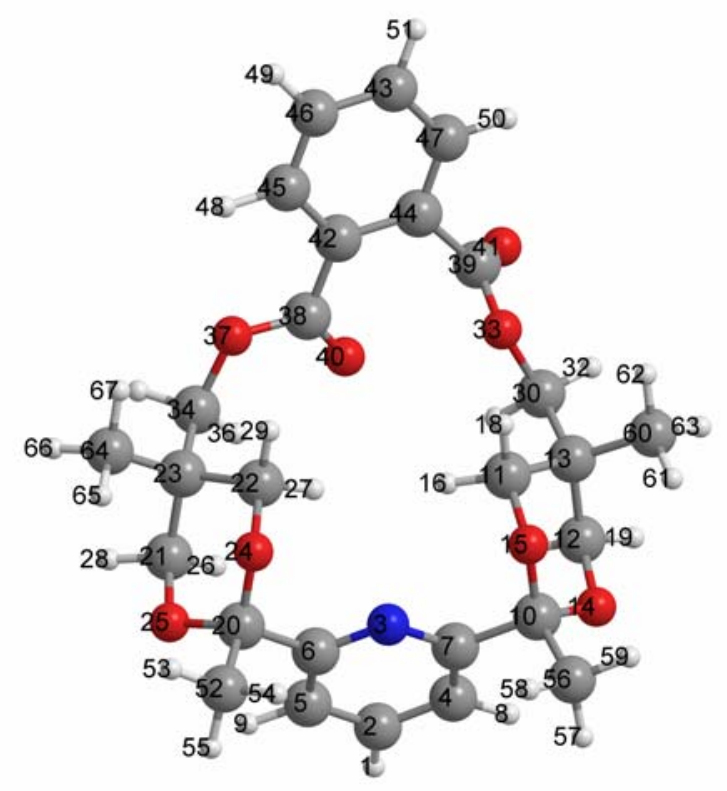

$\begin{array}{lrrr}\text { Atom } & \mathbf{X} & \mathbf{Y} & \mathbf{Z} \\ 1 & -4.741788 & 0.483944 & 2.784178 \\ 6 & -4.178083 & 0.403343 & 1.858229 \\ 7 & -2.743182 & 0.197806 & -0.515322 \\ 6 & -3.681930 & 1.546087 & 1.233724 \\ 6 & -3.936528 & -0.845182 & 1.286942 \\ 6 & -3.203299 & -0.899914 & 0.096473 \\ 6 & -2.964511 & 1.394321 & 0.041665 \\ 1 & -3.837880 & 2.535258 & 1.650632 \\ 1 & -4.298003 & -1.759212 & 1.745813 \\ 6 & -2.458027 & 2.628703 & -0.741836 \\ 6 & -0.172109 & 1.978769 & -0.882517 \\ 6 & -0.947685 & 3.473991 & 0.917080 \\ 6 & 0.274852 & 3.138127 & 0.031811 \\ 8 & -2.130495 & 3.696674 & 0.148807 \\ 8 & -1.370631 & 2.316040 & -1.577838 \\ 1 & -0.312947 & 1.062440 & -0.295897 \\ 1 & -1.117382 & 2.657366 & 1.636229 \\ 1 & 0.569846 & 1.775009 & -1.656092 \\ 1 & -0.791062 & 4.397654 & 1.484130 \\ 6 & -2.949100 & -2.245883 & -0.621643 \\ 6 & -1.693614 & -3.306359 & 1.123801 \\ 6 & -0.577960 & -2.079966 & -0.701398 \\ 6 & -0.400706 & -3.260167 & 0.274636 \\ 8 & -1.795550 & -2.204302 & -1.429827 \\ 8 & -2.871389 & -3.319565 & 0.319102 \\ 1 & -1.715156 & -2.442838 & 1.806661 \\ 1 & -0.552685 & -1.127134 & -0.157639\end{array}$




$\begin{array}{lrrr}1 & -1.743394 & -4.218442 & 1.727983 \\ 1 & 0.214423 & -2.066557 & -1.453128 \\ 6 & 1.413408 & 2.678255 & 0.951520 \\ 1 & 1.048549 & 2.039970 & 1.763736 \\ 1 & 1.947514 & 3.525618 & 1.391588 \\ 8 & 2.335885 & 1.874266 & 0.178904 \\ 6 & 0.770230 & -2.983655 & 1.231153 \\ 1 & 1.066422 & -3.896569 & 1.757854 \\ 1 & 0.515828 & -2.212975 & 1.960756 \\ 8 & 1.949311 & -2.541763 & 0.510791 \\ 6 & 2.396442 & -1.288173 & 0.773303 \\ 6 & 3.451489 & 1.474145 & 0.822245 \\ 8 & 1.856175 & -0.534004 & 1.560757 \\ 8 & 3.886439 & 1.979480 & 1.831588 \\ 6 & 3.631558 & -0.946925 & 0.010131 \\ 6 & 6.039362 & -0.320651 & -1.275967 \\ 6 & 4.144536 & 0.366279 & 0.071256 \\ 6 & 4.331013 & -1.927003 & -0.707007 \\ 6 & 5.524523 & -1.615190 & -1.354094 \\ 6 & 5.354395 & 0.661388 & -0.559687 \\ 1 & 3.934885 & -2.935130 & -0.742137 \\ 1 & 6.055955 & -2.383568 & -1.908002 \\ 1 & 5.755126 & 1.668194 & -0.490032 \\ 1 & 6.975406 & -0.073816 & -1.769091 \\ 6 & -4.110967 & -2.575476 & -1.552003 \\ 1 & -3.904564 & -3.522635 & -2.057875 \\ 1 & -4.216547 & -1.782152 & -2.296334 \\ 1 & -5.040033 & -2.664988 & -0.982575 \\ 6 & -3.560656 & 3.156733 & -1.654444 \\ 1 & -4.431382 & 3.457734 & -1.065590 \\ 1 & -3.852932 & 2.376288 & -2.361670 \\ 1 & -3.181126 & 4.021439 & -2.205521 \\ 6 & 0.698390 & 4.350228 & -0.812840 \\ 1 & -0.103154 & 4.640172 & -1.496558 \\ 1 & 1.588595 & 4.112031 & -1.406139 \\ 1 & 0.935580 & 5.210229 & -0.174556 \\ 6 & -0.196937 & -4.571192 & -0.499832 \\ 1 & -1.024497 & -4.739287 & -1.193240 \\ 1 & -0.142029 & -5.427324 & 0.184047 \\ 1 & 0.734253 & -4.537775 & -1.076762\end{array}$


Compound 3a-syn

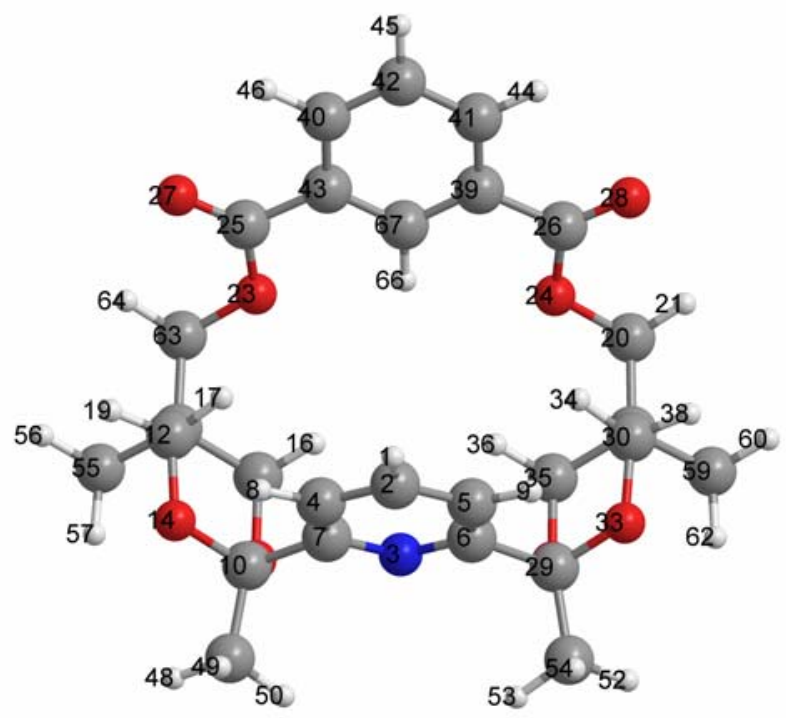

$\begin{array}{lrrr}\text { Atom } & \mathbf{X} & \mathbf{Y} & \mathbf{Z} \\ 1 & 3.266813 & -2.464227 & 0.000000 \\ 6 & 2.180351 & -2.430050 & 0.000000 \\ 7 & -0.605219 & -2.346570 & 0.000000 \\ 6 & 1.475042 & -2.413593 & 1.200928 \\ 6 & 1.475042 & -2.413593 & -1.200928 \\ 6 & 0.076902 & -2.368748 & -1.152595 \\ 6 & 0.076902 & -2.368748 & 1.152595 \\ 1 & 1.983046 & -2.448367 & 2.158081 \\ 1 & 1.983046 & -2.448367 & -2.158081 \\ 6 & -0.758222 & -2.485743 & 2.448092 \\ 6 & -1.881861 & -0.385055 & 2.347824 \\ 6 & 0.182819 & -0.636268 & 3.662104 \\ 6 & -1.161907 & 0.113530 & 3.616550 \\ 8 & -0.004833 & -2.051448 & 3.579248 \\ 8 & -1.992433 & -1.805668 & 2.361297 \\ 1 & -1.349989 & -0.051969 & 1.447170 \\ 1 & 0.829766 & -0.291236 & 2.843472 \\ 1 & -2.909492 & -0.009793 & 2.299093 \\ 1 & 0.705596 & -0.464318 & 4.608458 \\ 6 & -0.938894 & 1.630037 & -3.570385 \\ 1 & -0.382011 & 1.974019 & -4.445199 \\ 1 & -1.902706 & 2.150284 & -3.535315 \\ 8 & -0.225039 & 2.032019 & 2.379543 \\ 8 & -0.225039 & 2.032019 & -2.379543 \\ 6 & 0.948060 & 2.696288 & 2.527944 \\ 6 & 0.948060 & 2.696288 & -2.527944 \\ 8 & 1.447346 & 2.967441 & 3.601384 \\ 8 & 1.447346 & 2.967441 & -3.601384\end{array}$




$\begin{array}{lrrr}6 & -0.758222 & -2.485743 & -2.448092 \\ 6 & 0.182819 & -0.636268 & -3.662104 \\ 6 & -1.161907 & 0.113530 & -3.616550 \\ 8 & -1.992433 & -1.805668 & -2.361297 \\ 8 & -0.004833 & -2.051448 & -3.579248 \\ 1 & 0.829766 & -0.291236 & -2.843472 \\ 6 & -1.881861 & -0.385055 & -2.347824 \\ 1 & -1.349989 & -0.051969 & -1.447170 \\ 1 & -2.909492 & -0.009793 & -2.299093 \\ 1 & 0.705596 & -0.464318 & -4.608458 \\ 6 & 1.553749 & 3.048279 & -1.210804 \\ 6 & 2.774808 & 3.736966 & 1.207343 \\ 6 & 2.774808 & 3.736966 & -1.207343 \\ 6 & 3.380736 & 4.080386 & 0.000000 \\ 6 & 1.553749 & 3.048279 & 1.210804 \\ 1 & 3.230260 & 3.992245 & -2.158584 \\ 1 & 4.326017 & 4.615056 & 0.000000 \\ 1 & 3.230260 & 3.992245 & 2.158584 \\ 6 & -1.103580 & -3.950421 & 2.701290 \\ 1 & -1.701614 & -4.023350 & 3.613759 \\ 1 & -0.190327 & -4.539714 & 2.820082 \\ 1 & -1.679052 & -4.337559 & 1.856884 \\ 6 & -1.103580 & -3.950421 & -2.701290 \\ 1 & -1.701614 & -4.023350 & -3.613759 \\ 1 & -1.679052 & -4.337559 & -1.856884 \\ 1 & -0.190327 & -4.539714 & -2.820082 \\ 6 & -1.998900 & -0.198232 & 4.869547 \\ 1 & -1.483192 & 0.129188 & 5.780377 \\ 1 & -2.178599 & -1.272338 & 4.945812 \\ 1 & -2.971239 & 0.307947 & 4.829375 \\ 6 & -1.998900 & -0.198232 & -4.869547 \\ 1 & -1.483192 & 0.129188 & -5.780377 \\ 1 & -2.971239 & 0.307947 & -4.829375 \\ 1 & -2.178599 & -1.272338 & -4.945812 \\ 6 & -0.938894 & 1.630037 & 3.570385 \\ 1 & -0.382011 & 1.974019 & 4.445199 \\ 1 & -1.902706 & 2.150284 & 3.535315 \\ 1 & 0.000000 & 2.178419 & 0.000000 \\ 6 & 0.943239 & 2.707109 & 0.000000\end{array}$


Compound 3a-anti

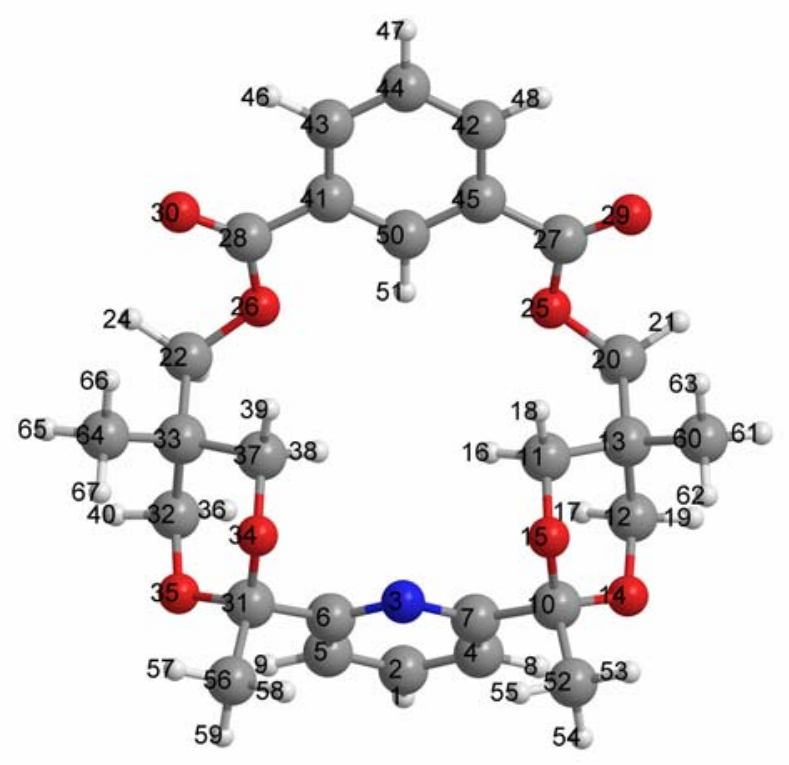

$\begin{array}{lrrr}\text { Atom } & \mathbf{X} & \mathbf{Y} & \mathbf{Z} \\ 1 & 4.300019 & -3.244731 & 0.000000 \\ 6 & 3.228700 & -3.060357 & 0.000000 \\ 7 & 0.484234 & -2.598035 & 0.000000 \\ 6 & 2.533575 & -2.944700 & 1.202322 \\ 6 & 2.533575 & -2.944700 & -1.202322 \\ 6 & 1.154873 & -2.709365 & -1.153725 \\ 6 & 1.154873 & -2.709365 & 1.153725 \\ 1 & 3.033676 & -3.044051 & 2.159386 \\ 1 & 3.033676 & -3.044051 & -2.159386 \\ 6 & 0.302423 & -2.687029 & 2.444268 \\ 6 & -0.502801 & -0.452310 & 2.265562 \\ 6 & 1.457760 & -0.960224 & 3.646842 \\ 6 & 0.233873 & -0.019501 & 3.552827 \\ 8 & 1.095657 & -2.337056 & 3.579530 \\ 8 & -0.817794 & -1.840617 & 2.326498 \\ 1 & 0.103190 & -0.237317 & 1.375999 \\ 1 & 2.163783 & -0.713532 & 2.837258 \\ 1 & -1.462021 & 0.059040 & 2.155405 \\ 1 & 1.981956 & -0.842339 & 4.601562 \\ 6 & 0.748811 & 1.443790 & 3.460225 \\ 1 & 0.509100 & 2.005635 & 4.364785 \\ 6 & 0.748811 & 1.443790 & -3.460225 \\ 1 & 1.831211 & 1.472609 & -3.298915 \\ 1 & 0.509100 & 2.005635 & -4.364785 \\ 8 & 0.206217 & 2.148694 & 2.322894 \\ 8 & 0.206217 & 2.148694 & -2.322894 \\ 6 & -0.802598 & 3.034017 & 2.515042\end{array}$




$\begin{array}{lrrr}6 & -0.802598 & 3.034017 & -2.515042 \\ 8 & -1.252510 & 3.334052 & 3.601964 \\ 8 & -1.252510 & 3.334052 & -3.601964 \\ 6 & 0.302423 & -2.687029 & -2.444268 \\ 6 & 1.457760 & -0.960224 & -3.646842 \\ 6 & 0.233873 & -0.019501 & -3.552827 \\ 8 & -0.817794 & -1.840617 & -2.326498 \\ 8 & 1.095657 & -2.337056 & -3.579530 \\ 1 & 2.163783 & -0.713532 & -2.837258 \\ 6 & -0.502801 & -0.452310 & -2.265562 \\ 1 & 0.103190 & -0.237317 & -1.375999 \\ 1 & -1.462021 & 0.059040 & -2.155405 \\ 1 & 1.981956 & -0.842339 & -4.601562 \\ 6 & -1.291068 & 3.573996 & -1.212166 \\ 6 & -2.302998 & 4.543419 & 1.209760 \\ 6 & -2.302998 & 4.543419 & -1.209760 \\ 6 & -2.799113 & 5.029803 & 0.000000 \\ 6 & -1.291068 & 3.573996 & 1.212166 \\ 1 & -2.690620 & 4.897639 & -2.159628 \\ 1 & -3.582118 & 5.782349 & 0.000000 \\ 1 & -2.690620 & 4.897639 & 2.159628 \\ 1 & 1.831211 & 1.472609 & 3.298915 \\ 6 & -0.782141 & 3.100479 & 0.000000 \\ 1 & 0.000000 & 2.355392 & 0.000000 \\ 6 & -0.246593 & -4.083353 & 2.720681 \\ 1 & -0.859840 & -4.052433 & 3.625327 \\ 1 & 0.573855 & -4.791645 & 2.864579 \\ 1 & -0.860432 & -4.404791 & 1.875715 \\ 6 & -0.246593 & -4.083353 & -2.720681 \\ 1 & -0.859840 & -4.052433 & -3.625327 \\ 1 & -0.860432 & -4.404791 & -1.875715 \\ 1 & 0.573855 & -4.791645 & -2.864579 \\ 6 & -0.688092 & -0.186177 & 4.773297 \\ 1 & -0.147761 & 0.035414 & 5.701858 \\ 1 & -1.070956 & -1.207101 & 4.835550 \\ 1 & -1.536794 & 0.503752 & 4.708448 \\ 6 & -0.688092 & -0.186177 & -4.773297 \\ 1 & -0.147761 & 0.035414 & -5.701858 \\ 1 & -1.536794 & 0.503752 & -4.708448 \\ 1 & -1.070956 & -1.207101 & -4.835550\end{array}$


Compound 4a (I).

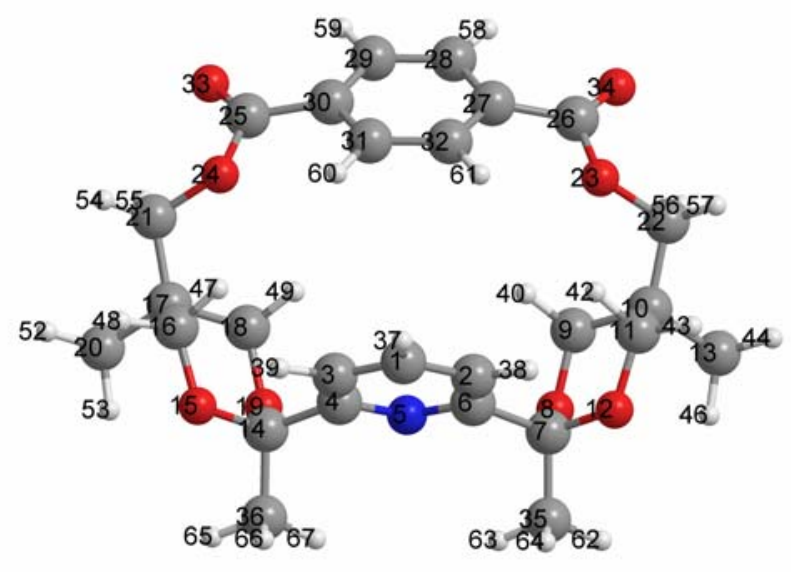

$\begin{array}{lrrc}\text { Atom } & \mathbf{X} & \mathbf{Y} & \mathbf{Z} \\ 6 & -0.000044 & -2.169771 & 2.628347 \\ 6 & 1.200197 & -2.198456 & 1.923511 \\ 6 & -1.200284 & -2.198433 & 1.923504 \\ 6 & -1.155553 & -2.221605 & 0.524016 \\ 7 & -0.000039 & -2.219601 & -0.158414 \\ 6 & 1.155469 & -2.221643 & 0.524018 \\ 6 & 2.445992 & -2.459436 & -0.302123 \\ 8 & 2.524395 & -1.663214 & -1.466768 \\ 6 & 2.820204 & -0.286250 & -1.241873 \\ 6 & 4.156911 & -0.131323 & -0.483257 \\ 6 & 3.997412 & -0.965997 & 0.799160 \\ 8 & 3.608595 & -2.310453 & 0.512897 \\ 6 & 5.331208 & -0.652563 & -1.328538 \\ 6 & -2.446073 & -2.459403 & -0.302118 \\ 8 & -3.608658 & -2.310367 & 0.512915 \\ 6 & -3.997438 & -0.965899 & 0.799145 \\ 6 & -4.156903 & -0.131235 & -0.483286 \\ 6 & -2.820208 & -0.286227 & -1.241909 \\ 8 & -2.524465 & -1.663209 & -1.466783 \\ 6 & -5.331229 & -0.652428 & -1.328552 \\ 6 & -4.440032 & 1.337246 & -0.128570 \\ 6 & 4.440101 & 1.337148 & -0.128546 \\ 8 & 3.291644 & 1.902768 & 0.536255 \\ 8 & -3.291555 & 1.902812 & 0.536234 \\ 6 & -2.846478 & 3.116555 & 0.121550 \\ 6 & 2.846574 & 3.116496 & 0.121520 \\ 6 & 1.394372 & 3.280142 & 0.423782\end{array}$




$\begin{array}{lrrr}6 & 0.695810 & 4.303586 & -0.230778 \\ 6 & -0.695695 & 4.303600 & -0.230768 \\ 6 & -1.394269 & 3.280169 & 0.423798 \\ 6 & -0.693978 & 2.327504 & 1.177656 \\ 6 & 0.694070 & 2.327491 & 1.177647 \\ 8 & -3.518767 & 3.907186 & -0.505951 \\ 8 & 3.518860 & 3.907095 & -0.506026 \\ 6 & 2.458216 & -3.906625 & -0.789116 \\ 6 & -2.458332 & -3.906605 & -0.789067 \\ 1 & -0.000049 & -2.158691 & 3.715401 \\ 1 & 2.152051 & -2.247764 & 2.437919 \\ 1 & -2.152142 & -2.247727 & 2.437910 \\ 1 & 2.004623 & 0.189493 & -0.682562 \\ 1 & 2.867837 & 0.168823 & -2.236633 \\ 1 & 3.266585 & -0.483505 & 1.462901 \\ 1 & 4.946659 & -1.049594 & 1.339913 \\ 1 & 6.281782 & -0.544273 & -0.791595 \\ 1 & 5.410842 & -0.101752 & -2.273195 \\ 1 & 5.191245 & -1.709989 & -1.561207 \\ 1 & -3.266609 & -0.483415 & 1.462891 \\ 1 & -4.946697 & -1.049461 & 1.339882 \\ 1 & -2.004600 & 0.189486 & -0.682610 \\ 1 & -2.867824 & 0.168834 & -2.236676 \\ 1 & -5.410736 & -0.101737 & -2.273289 \\ 1 & -6.281817 & -0.543931 & -0.791676 \\ 1 & -5.191401 & -1.709906 & -1.561069 \\ 1 & -5.300743 & 1.412827 & 0.546744 \\ 1 & -4.656946 & 1.932835 & -1.019831 \\ 1 & 4.657043 & 1.932719 & -1.019812 \\ 1 & 5.300809 & 1.412706 & 0.546773 \\ 1 & 1.255791 & 5.041315 & -0.795862 \\ 1 & -1.255668 & 5.041342 & -0.795842 \\ 1 & -1.235913 & 1.552945 & 1.706457 \\ 1 & 1.235995 & 1.552921 & 1.706444 \\ 1 & 3.367506 & -4.077979 & -1.371272 \\ 1 & 1.581746 & -4.082485 & -1.416482 \\ 1 & 2.440169 & -4.591793 & 0.062875 \\ 1 & -3.367666 & -4.077980 & -1.371146 \\ 1 & -2.440215 & -4.591752 & 0.062941 \\ 1 & -1.581908 & -4.082477 & -1.416494\end{array}$




\section{Compound 4a (II)}

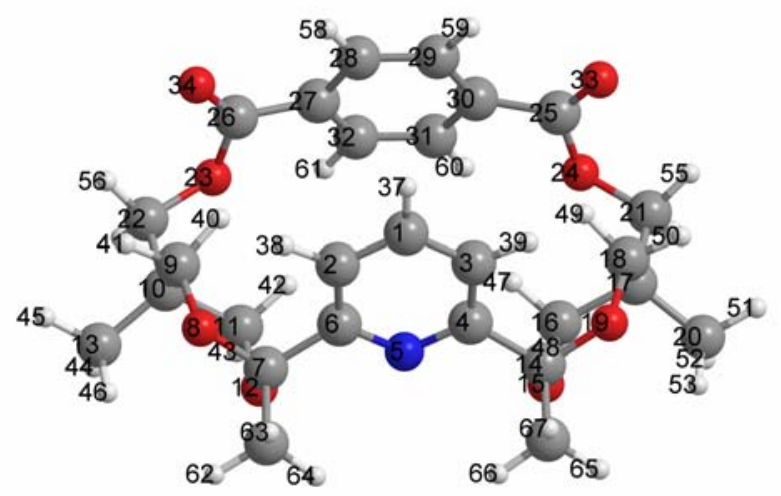

$\begin{array}{lccc}\text { Atom } & \mathbf{X} & \mathbf{Y} & \mathbf{Z} \\ 6 & 0.000072 & -1.195779 & 2.752485 \\ 6 & -1.199465 & -1.426680 & 2.085783 \\ 6 & 1.199539 & -1.426744 & 2.085681 \\ 6 & 1.154601 & -1.851834 & 0.752299 \\ 7 & -0.000066 & -2.047731 & 0.096762 \\ 6 & -1.154666 & -1.851769 & 0.752395 \\ 6 & -2.449881 & -2.316617 & 0.038269 \\ 8 & -3.606258 & -1.906768 & 0.766964 \\ 6 & -3.964465 & -0.529778 & 0.631407 \\ 6 & -4.138408 & -0.141093 & -0.849033 \\ 6 & -2.808389 & -0.537687 & -1.528744 \\ 8 & -2.526309 & -1.918943 & -1.316522 \\ 6 & -5.322745 & -0.909407 & -1.461866 \\ 6 & 2.449714 & -2.316694 & 0.038004 \\ 8 & 2.526081 & -1.918753 & -1.316721 \\ 6 & 2.808281 & -0.537484 & -1.528681 \\ 6 & 4.138415 & -0.141173 & -0.849021 \\ 6 & 3.964533 & -0.530109 & 0.631357 \\ 8 & 3.606170 & -1.907082 & 0.766705 \\ 6 & 5.322575 & -0.909567 & -1.462095 \\ 6 & 4.436720 & 1.367895 & -0.993317 \\ 6 & -4.436512 & 1.367965 & -0.993635 \\ 8 & -3.240802 & 2.169867 & -0.935640 \\ 8 & 3.241092 & 2.169926 & -0.935393 \\ 6 & 2.846613 & 2.701352 & 0.247686 \\ 6 & -2.846479 & 2.701487 & 0.247402 \\ 6 & -1.393827 & 3.042163 & 0.192303\end{array}$




$\begin{array}{lrrr}6 & -0.696365 & 3.202625 & 1.396651 \\ 6 & 0.696409 & 3.202590 & 1.396722 \\ 6 & 1.393986 & 3.042106 & 0.192442 \\ 6 & 0.693490 & 3.007212 & -1.022122 \\ 6 & -0.693211 & 3.007237 & -1.022191 \\ 8 & 3.552258 & 2.786972 & 1.231376 \\ 8 & -3.552267 & 2.787310 & 1.230970 \\ 6 & -2.481224 & -3.843261 & 0.022038 \\ 6 & 2.480906 & -3.843341 & 0.021497 \\ 1 & 0.000125 & -0.869286 & 3.789288 \\ 1 & -2.151942 & -1.321697 & 2.590484 \\ 1 & 2.152058 & -1.321831 & 2.590317 \\ 1 & -3.212919 & 0.112527 & 1.108767 \\ 1 & -4.901663 & -0.416833 & 1.185913 \\ 1 & -1.985193 & 0.079133 & -1.147259 \\ 1 & -2.855141 & -0.410931 & -2.615223 \\ 1 & -5.391411 & -0.728951 & -2.541285 \\ 1 & -6.270486 & -0.597464 & -1.005070 \\ 1 & -5.206976 & -1.982765 & -1.301819 \\ 1 & 1.985192 & 0.079334 & -1.146972 \\ 1 & 2.854917 & -0.410498 & -2.615138 \\ 1 & 3.213109 & 0.112223 & 1.108880 \\ 1 & 4.901786 & -0.417387 & 1.185814 \\ 1 & 6.270285 & -0.598666 & -1.004528 \\ 1 & 5.391855 & -0.728031 & -2.541294 \\ 1 & 5.206085 & -1.983020 & -1.303217 \\ 1 & 4.855721 & 1.573254 & -1.981953 \\ 1 & 5.142100 & 1.707428 & -0.228731 \\ 1 & -5.142008 & 1.707688 & -0.229241 \\ 1 & -4.855301 & 1.573186 & -1.982393 \\ 1 & -1.256209 & 3.247140 & 2.325157 \\ 1 & 1.256160 & 3.247073 & 2.325285 \\ 1 & 1.237656 & 2.913053 & -1.953846 \\ 1 & -1.237289 & 2.913095 & -1.953969 \\ 1 & -3.394633 & -4.175717 & -0.478389 \\ 1 & -2.467118 & -4.231541 & 1.044084 \\ 1 & -1.609374 & -4.215414 & -0.520533 \\ 1 & 3.394254 & -4.175795 & -0.479042 \\ 1 & 1.608986 & -4.215306 & -0.521088 \\ 1 & 2.466825 & -4.231807 & 1.043473\end{array}$




\section{Compound 5a-syn}

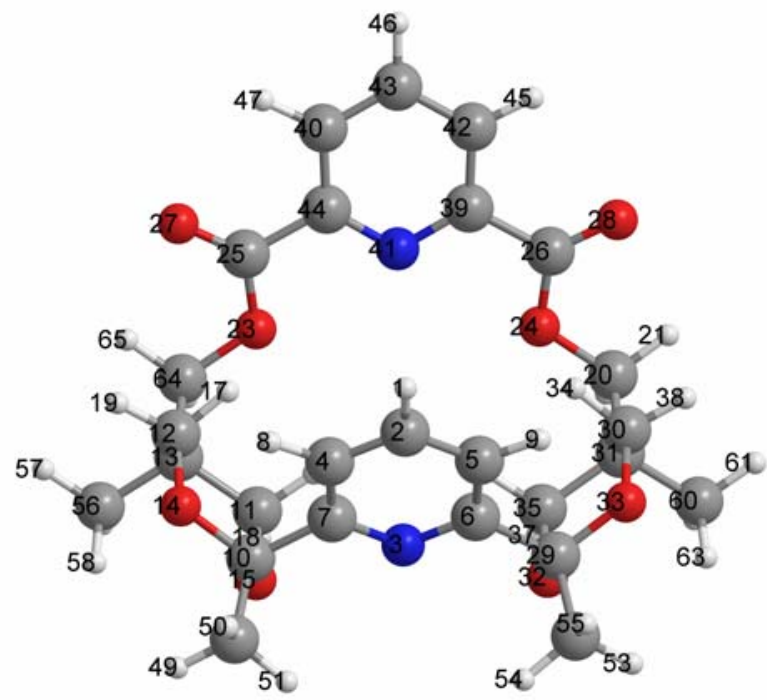

$\begin{array}{lrrr}\text { Atom } & \mathbf{X} & \mathbf{Y} & \mathbf{Z} \\ 1 & -3.776782 & 1.550953 & 0.000000 \\ 6 & -2.716182 & 1.789522 & 0.000000 \\ 7 & 0.000000 & 2.407918 & 0.000000 \\ 6 & -2.028784 & 1.947829 & 1.201344 \\ 6 & -2.028784 & 1.947829 & -1.201344 \\ 6 & -0.664149 & 2.255056 & -1.152227 \\ 6 & -0.664149 & 2.255056 & 1.152227 \\ 1 & -2.527193 & 1.848426 & 2.159165 \\ 1 & -2.527193 & 1.848426 & -2.159165 \\ 6 & 0.122134 & 2.561344 & 2.446913 \\ 6 & 1.760690 & 0.836805 & 2.283873 \\ 6 & -0.282010 & 0.499352 & 3.613238 \\ 6 & 1.213552 & 0.136361 & 3.542927 \\ 8 & -0.476975 & 1.915728 & 3.570363 \\ 8 & 1.490461 & 2.236093 & 2.336676 \\ 1 & 1.327735 & 0.392163 & 1.378765 \\ 1 & -0.822648 & 0.021568 & 2.784593 \\ 1 & 2.850567 & 0.750195 & 2.222013 \\ 1 & -0.732274 & 0.167114 & 4.553837 \\ 6 & 1.408818 & -1.382838 & -3.455003 \\ 1 & 1.005161 & -1.882863 & -4.338585 \\ 1 & 2.474286 & -1.618084 & -3.363139 \\ 8 & 0.790649 & -1.944800 & 2.273879 \\ 8 & 0.790649 & -1.944800 & -2.273879 \\ 6 & -0.264971 & -2.756159 & 2.446550\end{array}$




$\begin{array}{lrrr}6 & -0.264971 & -2.756159 & -2.446550 \\ 8 & -0.740218 & -3.065520 & 3.522402 \\ 8 & -0.740218 & -3.065520 & -3.522402 \\ 6 & 0.122134 & 2.561344 & -2.446913 \\ 6 & -0.282010 & 0.499352 & -3.613238 \\ 6 & 1.213552 & 0.136361 & -3.542927 \\ 8 & 1.490461 & 2.236093 & -2.336676 \\ 8 & -0.476975 & 1.915728 & -3.570363 \\ 1 & -0.822648 & 0.021568 & -2.784593 \\ 6 & 1.760690 & 0.836805 & -2.283873 \\ 1 & 1.327735 & 0.392163 & -1.378765 \\ 1 & 2.850567 & 0.750195 & -2.222013 \\ 1 & -0.732274 & 0.167114 & -4.553837 \\ 6 & -0.822563 & -3.261017 & -1.142575 \\ 6 & -1.878411 & -4.180493 & 1.197870 \\ 7 & -0.298284 & -2.807438 & 0.000000 \\ 6 & -1.878411 & -4.180493 & -1.197870 \\ 6 & -2.411201 & -4.649148 & 0.000000 \\ 6 & -0.822563 & -3.261017 & 1.142575 \\ 1 & -2.252627 & -4.500582 & -2.163476 \\ 1 & -3.228728 & -5.364182 & 0.000000 \\ 1 & -2.252627 & -4.500582 & 2.163476 \\ 6 & 0.068973 & 4.058885 & 2.736301 \\ 1 & 0.637091 & 4.266223 & 3.647277 \\ 1 & -0.966181 & 4.382534 & 2.874806 \\ 1 & 0.511645 & 4.604914 & 1.899557 \\ 6 & 0.068973 & 4.058885 & -2.736301 \\ 1 & 0.637091 & 4.266223 & -3.647277 \\ 1 & 0.511645 & 4.604914 & -1.899557 \\ 1 & -0.966181 & 4.382534 & -2.874806 \\ 6 & 1.950299 & 0.628002 & 4.801156 \\ 1 & 1.542957 & 0.159934 & 5.705544 \\ 1 & 1.847375 & 1.710280 & 4.900218 \\ 1 & 3.020194 & 0.391224 & 4.748829 \\ 6 & 1.950299 & 0.628002 & -4.801156 \\ 1 & 1.542957 & 0.159934 & -5.705544 \\ 1 & 3.020194 & 0.391224 & -4.748829 \\ 1 & 1.847375 & 1.710280 & -4.900218 \\ 6 & 1.408818 & -1.382838 & 3.455003 \\ 1 & 1.005161 & -1.882863 & 4.338585 \\ 1 & 2.474286 & -1.618084 & 3.363139\end{array}$




\section{Compound 5a-anti}

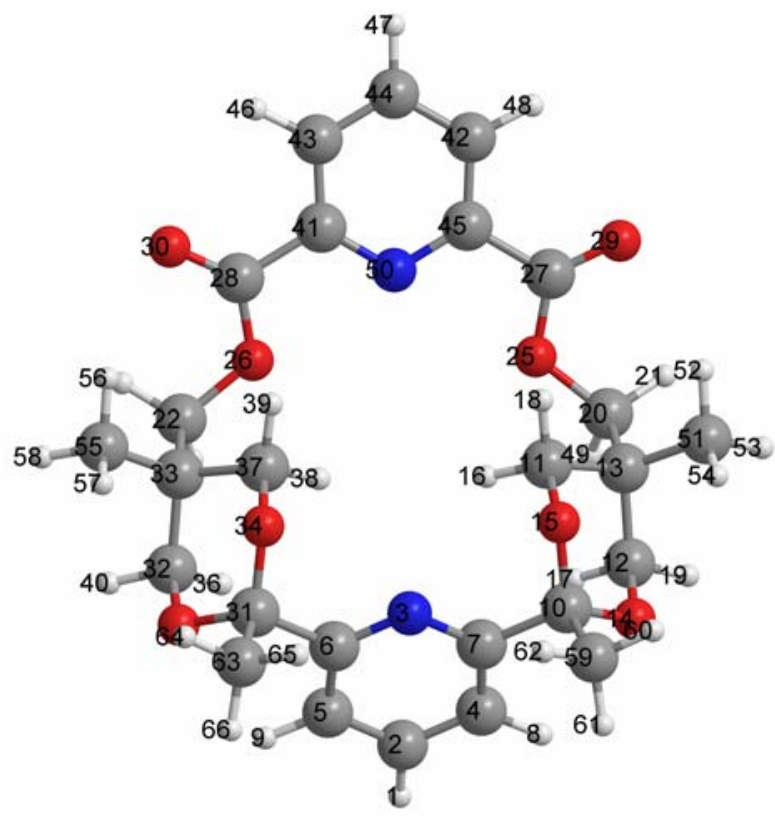

$\begin{array}{lrrr}\text { Atom } & \mathbf{X} & \mathbf{Y} & \mathbf{Z} \\ 1 & -3.601684 & 4.080509 & 0.000000 \\ 6 & -2.590877 & 3.680443 & 0.000000 \\ 7 & 0.000000 & 2.663300 & 0.000000 \\ 6 & -1.934867 & 3.422810 & 1.202130 \\ 6 & -1.934867 & 3.422810 & -1.202130 \\ 6 & -0.634357 & 2.908130 & -1.153176 \\ 6 & -0.634357 & 2.908130 & 1.153176 \\ 1 & -2.404856 & 3.618460 & 2.159646 \\ 1 & -2.404856 & 3.618460 & -2.159646 \\ 6 & 0.189407 & 2.701643 & 2.445519 \\ 6 & 0.569677 & 0.358682 & 2.217681 \\ 6 & -1.286303 & 1.197925 & 3.587444 \\ 6 & -0.261088 & 0.045533 & 3.483112 \\ 8 & -0.666976 & 2.481198 & 3.567956 \\ 8 & 1.133437 & 1.665114 & 2.315244 \\ 1 & -0.041588 & 0.267384 & 1.311571 \\ 1 & -2.009444 & 1.114375 & 2.760265 \\ 1 & 1.422611 & -0.316533 & 2.117196 \\ 1 & -1.842547 & 1.155859 & 4.530428 \\ 6 & -1.041612 & -1.289026 & 3.338754 \\ 1 & -0.963746 & -1.892338 & 4.245378 \\ 6 & -1.041612 & -1.289026 & -3.338754 \\ 1 & -2.097831 & -1.107373 & -3.117545 \\ 1 & -0.963746 & -1.892338 & -4.245378 \\ 8 & -0.587647 & -2.073783 & 2.213366 \\ 8 & -0.587647 & -2.073783 & -2.213366 \\ 6 & 0.245806 & -3.101736 & 2.434182\end{array}$




$\begin{array}{lrrr}6 & 0.245806 & -3.101736 & -2.434182 \\ 8 & 0.628634 & -3.475415 & 3.525621 \\ 8 & 0.628634 & -3.475415 & -3.525621 \\ 6 & 0.189407 & 2.701643 & -2.445519 \\ 6 & -1.286303 & 1.197925 & -3.587444 \\ 6 & -0.261088 & 0.045533 & -3.483112 \\ 8 & 1.133437 & 1.665114 & -2.315244 \\ 8 & -0.666976 & 2.481198 & -3.567956 \\ 1 & -2.009444 & 1.114375 & -2.760265 \\ 6 & 0.569677 & 0.358682 & -2.217681 \\ 1 & -0.041588 & 0.267384 & -1.311571 \\ 1 & 1.422611 & -0.316533 & -2.117196 \\ 1 & -1.842547 & 1.155859 & -4.530428 \\ 6 & 0.668145 & -3.755106 & -1.144178 \\ 6 & 1.467392 & -4.903718 & 1.200376 \\ 6 & 1.467392 & -4.903718 & -1.200376 \\ 6 & 1.868201 & -5.487132 & 0.000000 \\ 6 & 0.668145 & -3.755106 & 1.144178 \\ 1 & 1.756001 & -5.305605 & -2.165090 \\ 1 & 2.487684 & -6.379252 & 0.000000 \\ 1 & 1.756001 & -5.305605 & 2.165090 \\ 1 & -2.097831 & -1.107373 & 3.117545 \\ 7 & 0.272962 & -3.191283 & 0.000000 \\ 6 & 0.651403 & 0.005833 & 4.721492 \\ 1 & 1.350803 & -0.834841 & 4.656567 \\ 1 & 0.059894 & -0.121862 & 5.636584 \\ 1 & 1.223584 & 0.931888 & 4.810895 \\ 6 & 0.651403 & 0.005833 & -4.721492 \\ 1 & 1.350803 & -0.834841 & -4.656567 \\ 1 & 1.223584 & 0.931888 & -4.810895 \\ 1 & 0.059894 & -0.121862 & -5.636584 \\ 6 & 0.985712 & 3.964982 & 2.757215 \\ 1 & 1.572298 & 3.800576 & 3.665169 \\ 1 & 0.310541 & 4.811105 & 2.911389 \\ 1 & 1.658460 & 4.183660 & 1.924274 \\ 6 & 0.985712 & 3.964982 & -2.757215 \\ 1 & 1.572298 & 3.800576 & -3.665169 \\ 1 & 1.658460 & 4.183660 & -1.924274 \\ 1 & 0.310541 & 4.811105 & -2.911389\end{array}$

